\title{
Nanomechanical Characterization of Amyloid Fibrils Using Single-Molecule Experiments and Computational Simulations
}

\author{
Bumjoon Choi, ${ }^{1}$ Taehee Kim, ${ }^{2}$ Sang Woo Lee, ${ }^{1}$ and Kilho Eom ${ }^{3}$ \\ ${ }^{1}$ Department of Biomedical Engineering, Yonsei University, Wonju 26493, Republic of Korea \\ ${ }^{2}$ College of Sport Science, Sungkyunkwan University (SKKU), Suwon 16419, Republic of Korea \\ ${ }^{3}$ Biomechanics Laboratory, College of Sport Science, Sungkyunkwan University (SKKU), Suwon 16419, Republic of Korea
}

Correspondence should be addressed to Kilho Eom; kilhoeom@skku.edu

Received 11 February 2016; Revised 29 April 2016; Accepted 5 May 2016

Academic Editor: Silvia Licoccia

Copyright (C) 2016 Bumjoon Choi et al. This is an open access article distributed under the Creative Commons Attribution License, which permits unrestricted use, distribution, and reproduction in any medium, provided the original work is properly cited.

\begin{abstract}
Amyloid fibrils have recently received much attention due to not only their important role in disease pathogenesis but also their excellent mechanical properties, which are comparable to those of mechanically strong protein materials such as spider silk. This indicates the necessity of understanding fundamental principles providing insight into how amyloid fibrils exhibit the excellent mechanical properties, which may allow for developing biomimetic materials whose material (e.g., mechanical) properties can be controlled. Here, we describe recent efforts to characterize the nanomechanical properties of amyloid fibrils using computational simulations (e.g., atomistic simulations) and single-molecule experiments (e.g., atomic force microscopy experiments). This paper summarizes theoretical models, which are useful in analyzing the mechanical properties of amyloid fibrils based on simulations and experiments, such as continuum elastic (beam) model, elastic network model, and polymer statistical model. In this paper, we suggest how the nanomechanical properties of amyloid fibrils can be characterized and determined using computational simulations and/or atomic force microscopy experiments coupled with the theoretical models.
\end{abstract}

\section{Introduction}

Amyloid fibrils, which are formed by self-assembly process (i.e., protein aggregation) [1-4], have recently received significant attention due to not only their important role in disease pathologies [5-9] but also their excellent nanomechanical properties [10]. In particular, amyloid materials have been found to be associated with various diseases such as neurodegenerative diseases [5-7] (e.g., Alzheimer's disease and Parkinson's disease), type II diabetes [8], and cardiovascular disease [11]. These amyloid materials exhibit multiscale structural feature [10] such that they are amyloid small aggregate (e.g., amyloid oligomer) at nanoscale, amyloid fibril at submicron scale, and amyloid plaques at micron scale. More remarkably, amyloid materials such as oligomers and fibrils are the ordered structures made of $\beta$-strands [1]. This ordered structure of amyloid materials is responsible for their insolubility in physiological condition and also for their remarkable (mechanical) properties. Specifically, $\beta$ strand-rich protein structures (e.g., amyloid) are stable due to hydrogen bonds that sustain (and stabilize) $\beta$-sheet-rich structures $[12,13]$. In addition, recent experimental studies $[14,15]$ report that the mechanical properties of amyloid fibril are determined from the content of $\beta$-sheets comprising the fibril in such a way that as the $\beta$-sheet content increases the mechanical properties (e.g., elastic modulus) of the fibril are improved.

The nanomechanical properties of amyloid fibrils have recently been measured based on experimental and computational techniques. In particular, atomic force microscopy (AFM) or cryo-electron microscopy (EM) imaging techniques coupled with polymer chain statistics have allowed for estimating the bending property (i.e., persistent length) of amyloid fibrils [16, 17]. Specifically, the mechanical properties of protein fibrils are measured based on their fluctuation behavior, which can be obtained from AFM images or cryoEM images. Recently, this imaging-based mechanical characterization has been implemented in FiberApp [18], which is an open software for analyzing the structures and mechanical 
properties of protein fibrils based on AFM images. Moreover, AFM nanoindentation technique has enabled the mechanical characterization of amyloid fibrils [19-21]. In addition, computational simulations based on atomistic and/or elastic network models have been extensively employed for characterizing the nanomechanical properties of amyloid fibrils [10, 22-24]. Based on experimental and computational techniques, the elastic modulus of amyloid fibrils is measured in the order of 1 to $10 \mathrm{GPa}[10,22-24]$, which is comparable to that of a spider silk protein [25], which is known as one of the mechanically strong proteins. Moreover, the toughness of amyloid small aggregates with their size of $\sim 3 \mathrm{~nm}$ is measured as $\sim 30 \mathrm{kcal} \cdot \mathrm{mol}^{-1} \cdot \mathrm{nm}^{-3}$ [23], which is very close to that of spider silk crystal with its size of $\sim 2 \mathrm{~nm}$. Recent experimental [26] and computational studies $[22,23]$ report an important role that the length scale of protein fibrils plays in their nanomechanical properties. This length scale effect in the nanomechanical properties of amyloid fibrils is attributed to the fact that the deformation mechanism of the fibrils depends on their length scale.

The remarkable mechanical properties of amyloid fibrils have recently been found to play a pivotal role in their biological functions such as disease pathologies [36]. For instance, it has recently been reported that the mechanical disruption of cell membrane due to amyloid fibril [37] is ascribed to the fact that the elastic modulus of amyloid fibrils is in the order of 1 to $10 \mathrm{GPa}$ [10], whereas the elastic modulus of cell membrane is in the order of $100 \mathrm{kPa}$ [38]. This observation suggests an evidence that the nanomechanical properties of amyloid fibrils are highly correlated with their biological functions. Moreover, a recent study by Tanaka and coworkers [39] reports that prion infectivity is determined from the fracture toughness (brittleness) of prion fibrils such that the softer the fibril, the higher the prion infectivity. In addition, as described in our recent study [30], the sizedependent elastic properties of HET-s prion fibrils provide insight into their critical size related to prion infectivity. These observations highlight a role that the nanomechanical properties of amyloid fibrils play in their pathological functions.

In this paper, we describe recent attempts to characterize the nanomechanical properties of amyloid fibrils by using experiments and simulations, as the mechanical characterization of the fibrils is of great importance for further understanding of their biological functions. This paper is organized as follows: Section 2 summarizes theoretical models such as elastic network model, elastic beam model, and polymer chin model, which can be coupled with experiments or computational simulations in order to measure the mechanical properties of amyloid fibrils. In Section 3, we provide the recent efforts to characterize the nanomechanical properties of amyloid fibrils with using single-molecule experiments based on atomic force microscopy (AFM). Section 4 is dedicated to the review of computational efforts to measure the mechanical properties of amyloid fibrils as well as gain insight into their deformation mechanisms. In Section 5, we conclude this paper with providing remarks and further directions that can be considered for future works.

\section{Theoretical Models}

In this section, we summarize theoretical models that are useful in analyzing the experimental and simulation results to extract the nanomechanical properties of amyloid fibrils. Here, we note that the principles of computational simulations such as atomistic simulation and normal mode analysis are not included in this review, but the principles of these simulations including steered molecular dynamics (SMD) simulations are well described in [40-44]. In addition, though we describe recent experimental attempts to characterize the mechanical properties of amyloid fibrils, we skip the details of AFM experimental methods (e.g., AFM imaging and indentation), which are well summarized in [45]. In the following, we provide theoretical models such as polymer chain model, elastic network model, and continuum elastic beam model.

2.1. Polymer Chain Model: Polymer Chain Statistics. Polymer chain models have allowed for understanding the fluctuation behavior of one-dimensional polymer molecules [46]. Among polymer chain models, a wormlike chain (WLC) model [47] has been widely accepted for analyzing the fluctuation behavior of a biological molecule $[48,49]$ such as DNA chain $[50,51]$ and protein fibril, for example, microtubule [52]. The WLC model assumes that a biological fibril is composed of rigid subchains. The energy $(V)$ required to bend an angle $(\theta)$ between two subchains located a distance $\xi$ apart is given by

$$
V=\frac{E_{B} I}{2 \xi} \theta^{2}
$$

where $E_{B}$ and $I$ are elastic modulus and cross-sectional moment of inertia for a biological fibril, respectively. A probability distribution function for an angle $\theta$ obeys Boltzmann's distribution such as

$$
p(\theta)=\sqrt{\frac{E_{B} I}{2 \xi k_{B} T}} \exp \left(-\frac{E_{B} I}{2 \xi k_{B} T} \theta^{2}\right),
$$

where $k_{B}$ and $T$ are Boltzmann's constant and absolute temperature, respectively. Here, it should be noted that the persistent length $\left(L_{P}\right)$ of a biological fibril is defined as $L_{P}=$ $E_{B} I / k_{B} T$. The ensemble averages for an angle $\theta$ and its cosine value are given by [49]

$$
\begin{aligned}
\left\langle\theta^{2}\right\rangle & =\frac{\xi}{L_{P}}, \\
\langle\cos \theta\rangle & =\exp \left(-\frac{\xi}{2 L_{P}}\right) .
\end{aligned}
$$

The mean-squared value of the end-to-end distance $(R)$ of a biological fibril in a two-dimensional space (i.e., on the surface) is represented in the form of [51]

$$
\frac{\left\langle R^{2}\right\rangle}{L^{2}}=4\left(\frac{L}{L_{P}}\right)\left[1-\frac{2 L_{P}}{L}\left\{1-\exp \left(-\frac{L}{2 L_{P}}\right)\right\}\right],
$$

where $L$ is a contour length of a biological fibril. 
2.2. Elastic Network Model. Coarse-grained (CG) model enables not only the computationally efficient dynamic analysis of protein molecules [41,53-56] but also the insight into a role that the structure of protein molecules plays in their dynamics and mechanics. The computational efficacy of CG model is due to the modeling strategy in that minimal degrees of freedom are used to model a protein structure with simplified interaction potential field.

Among CG models, an elastic network model (ENM) $[41,57-64]$ is not only a most simple and computationally efficient model, but also a minimalist model which can provide an insight into the role of protein structure on the mechanics and dynamics of a protein molecule. The basic idea of ENM is to consider only the $\alpha$-carbon atoms of a protein structure, while interaction between these $\alpha$-carbon atoms is simplified as harmonic interaction. The potential field of ENM is given by

$$
V=\frac{\gamma}{2} \sum_{i, j}\left(\left|\vec{r}_{i}-\vec{r}_{j}\right|-\left|\vec{r}_{i}^{0}-\vec{r}_{j}^{0}\right|\right)^{2} \cdot H\left(R_{c}-\left|\vec{r}_{i}^{0}-\vec{r}_{j}^{0}\right|\right),
$$

where $\gamma$ is the force constant of a harmonic potential defined between two $\alpha$-carbon atoms $i$ and $j, \vec{r}_{i}$ is the position vector of the $i$ th $\alpha$-carbon atom, superscript 0 indicates the equilibrium state, $R_{c}$ is a cut-off distance (that is typically defined as $R_{c}=10 \sim 14 \AA$ ), and $H(x)$ is a Heaviside unit step function defined as $H(x)=1$ if $x>0$; otherwise, $H(x)=$ 0 . This ENM coupled with normal mode analysis is able to provide the elastic properties of amyloid fibrils, which is well described in $[22,27]$.

2.3. Continuum Model: Elastic Beam Model. Amyloid fibrils can be modeled as a one-dimensional beam model due to their structural feature in that their length is much larger than their thickness. Here, we consider the vibrational and deformation behaviors of the fibril based on Euler-Bernoulli beam theory. In addition, we present the Timoshenko beam theory useful for understanding the length-dependent mechanical properties of amyloid fibrils.

2.3.1. Vibrational Behavior of Fibril Structure. Let us consider the vibrational (bending) motion of amyloid fibril. The equation of motion for a fibril modeled based on EulerBernoulli beam [65] is given by

$$
\rho A \partial_{t}^{2} w(x, t)+\partial_{x}^{2}\left[E I \partial_{x}^{2} w(x, t)\right]=0,
$$

where $\rho, A, E$, and $I$ represent the density, cross-sectional area, elastic modulus, and cross-sectional moment of inertia of the fibril, respectively, $w(x, t)$ is its transverse deflection, and a coordinate $x$ is defined along the fibril axis. With assuming $w(x, t)=u(x) \cdot \exp [j \omega t]$, the equation of motion given by (7) becomes an eigenvalue problem as follows:

$$
\omega^{2} \rho A u=E I \partial_{x}^{4} u .
$$

Consequently, the frequency of the fibril is represented in the form of

$$
\omega=\left(\frac{\lambda}{L}\right)^{2} \sqrt{\frac{E I}{\rho A}}
$$

where $L$ is the length of an amyloid fibril and $\lambda$ is a boundary condition-dependent constant. Equation (9) clearly demonstrates that it is straightforward to compute the elastic modulus (or equivalently, bending rigidity) of an amyloid fibril if its natural frequency was measured from computational simulations (e.g., atomistic simulations or ENM simulations) or experiments (based on 4D electron microscopy). In addition, the torsional shear modulus and axial elastic modulus of amyloid fibrils can be also characterized based on measuring their natural frequencies corresponding to torsional (twisting) and axial stretching deformation modes, respectively. The details of extracting these torsional shear modulus and axial elastic modulus based on the elastic beam model are well described in $[22,27]$.

2.3.2. Mechanical (Bending) Deformation of Fibril. Let us take into account the force-driven (bending) deformation of an amyloid fibril. The governing equation for the bending deformation of a fibril due to a force is given by [66]

$$
\partial_{x}^{2}\left[E I \partial_{x}^{2} w(x)\right]=q(x)
$$

where $q(x)$ is a distributed load acting on the fibril. In case of a force, $F$, acting on the specific location $\left(x=x_{0}\right)$ of the fibril, the governing equation given by (11) becomes the following equation:

$$
\partial_{x}^{2}\left[E I \partial_{x}^{2} w(x)\right]=F \cdot \delta\left(x-x_{0}\right)
$$

Here, $\delta(x)$ is a Dirac delta function. Consequently, the forcedisplacement relationship of deformed fibril is given by

$$
W=\frac{F L^{3}}{a E I}
$$

where $W$ is displacement at the location where a force is applied; that is, $W=w\left(x_{0}\right) ; a$ is a constant that depends on boundary condition and the location at which a force is applied. It is straightforward to extract the bending rigidity (or bending elastic modulus) of an amyloid fibril, if its forcedisplacement relationship (i.e., $F$ versus $W$ ) is obtained from simulation or experiment.

2.3.3. Timoshenko Beam Model: Length-Dependent Property. For understanding the effect of fibril length on the bending properties of protein fibril, recent studies [22, 23, 27, 30, 52, 67] have employed the Timoshenko beam theory [68], which assumes that the deformation of the fibril is attributed to both shear and bending deformations. The total deflection of the fibril is represented in the form of

$$
W=\frac{F L^{3}}{a E I}+\frac{c F L}{b G_{S} A}
$$

where $G_{S}$ and $A$ represent the shear modulus and crosssectional area of an amyloid fibril, respectively, $b$ is a boundary condition-dependent constant, and $c$ is a shear coefficient that depends on the cross-sectional shape of an amyloid fibril. 


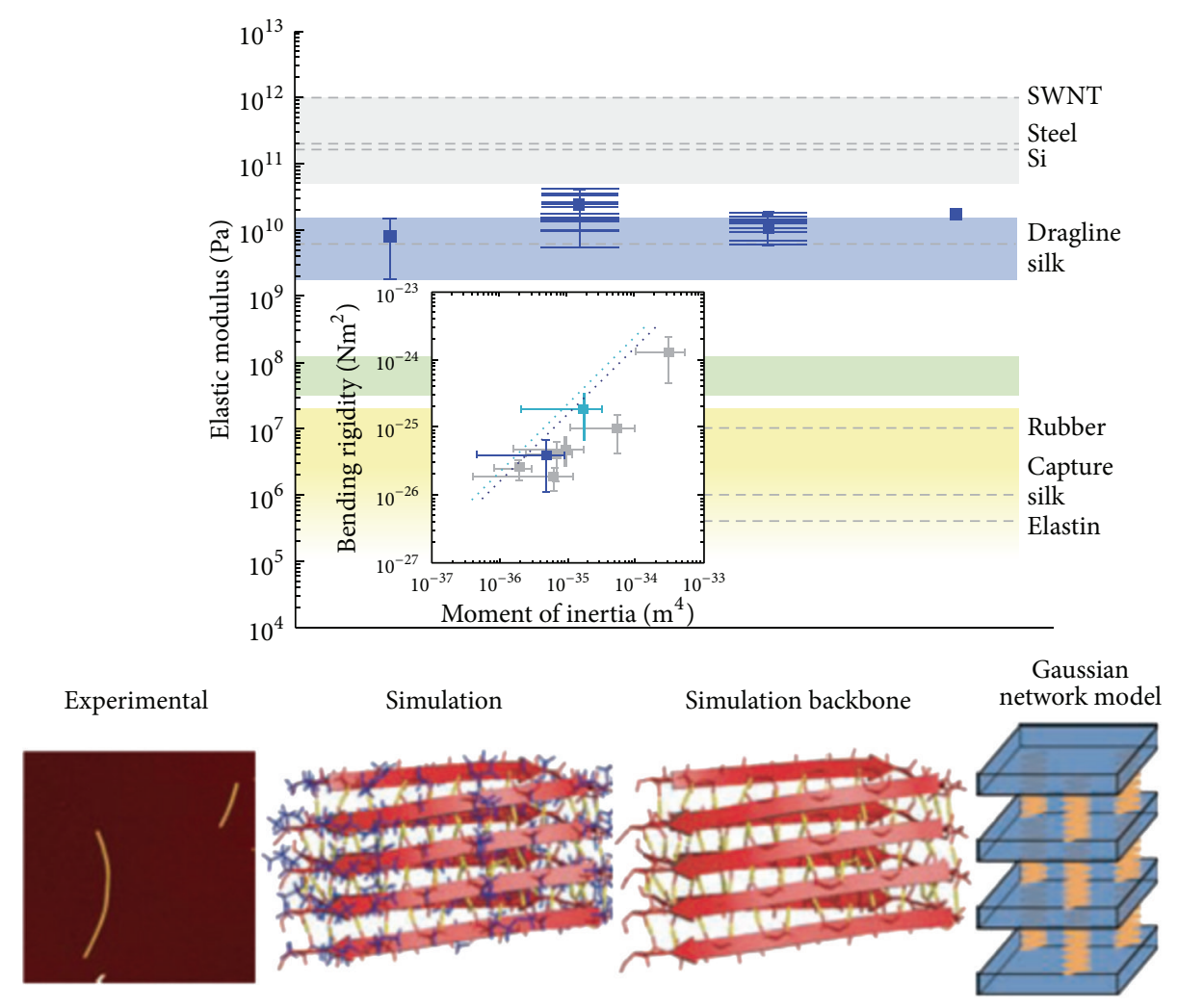

FIGURE 1: Nanomechanical properties of amyloid fibrils and the role of intermolecular forces in the nanomechanical properties. The elastic modulus of amyloid fibrils is measured in a range of 1 to $10 \mathrm{GPa}$ (blue region in the graph), and this elastic modulus is comparable to that of dragline silk. The nanomechanical properties of amyloid fibrils are measured based on AFM-based single-molecule experiments and simulations such as full-atomistic simulations, backbone-based atomistic simulations, and elastic network model (as shown in figures from lower left panel to lower right panel, resp.). Figures are adopted with permission from [32].

From (13), the effective bending rigidity of an amyloid fibril is given by

$$
\widetilde{E} I=E I\left(1+\frac{a}{b} \frac{c E I}{G_{S} A L^{2}}\right)^{-1} .
$$

The Timoshenko beam theory given by (14) provides the length-dependent bending rigidity of an amyloid fibril, which is ascribed to competition between shear and bending deformations.

\section{Single-Molecule Force Spectroscopy-Based Mechanical Characterization of Amyloid Fibrils}

Single-molecule force spectroscopy based on optical tweezer or AFM is a useful experimental toolkit that allows for mechanical characterization of protein materials at molecular scale [69-72]. For a recent decade, single-molecule force spectroscopy has been utilized to probe the mechanical properties of amyloid fibrils [10, 45]. Here, we briefly provide the recent key efforts to experimentally characterize the nanomechanical properties of amyloid fibrils using force spectroscopy based on AFM.

In recent years, Knowles and coworkers [32] have reported the nanomechanical properties of amyloid fibrils using AFM imaging experiments coupled with polymer chain statistics that is described in Section 2.1. In particular, they measured the fluctuation of angle between two tangent vectors, which results in the extraction of mechanical properties such as persistent length-for example, see (3)from the AFM images of amyloid fibrils. The nanomechanical properties of several amyloid fibrils such as $\alpha$-lactalbumin fibril, insulin $\beta$-chain fibril, $\beta$-lactoglobulin fibril, insulin fibril, and TTR (105-115) fibril were measured using AFM imaging experiments coupled with polymer chain statistics. The bending elastic modulus of these fibrils is measured in a range of 1 to $10 \mathrm{GPa}$ (Figure 1). They found that, based on AFM experiments and simulations such as atomistic simulations and ENM, the excellent mechanical properties of these fibrils are attributed to intermolecular forces that act between $\beta$-sheet layers (Figure 1 ). In addition, they reported that the elastic modulus of amyloid fibril is close to that of mechanically strong spider silk protein and that the fracture property of amyloid fibril is related to the growth mechanisms of amyloid fibrils [26].

Recently, Adamcik and colleagues have reported the nanomechanical properties of multistranded amyloid fibrils using AFM imaging experiments together with polymer chain statistics [16] (Figure 2). They reported that amyloid fibrils can be formed as a multistranded fibril that is 


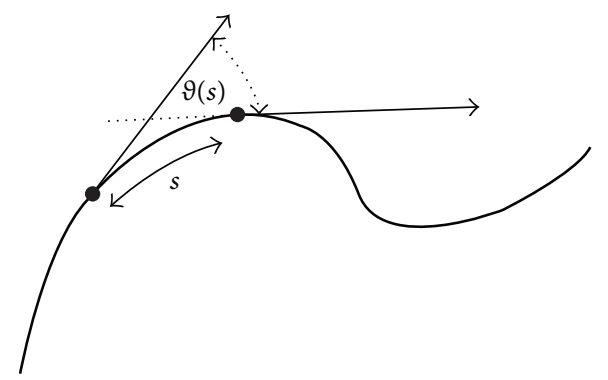

(a)

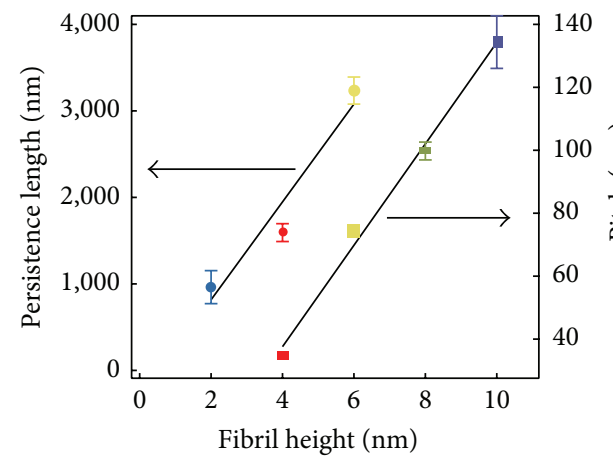

(c)

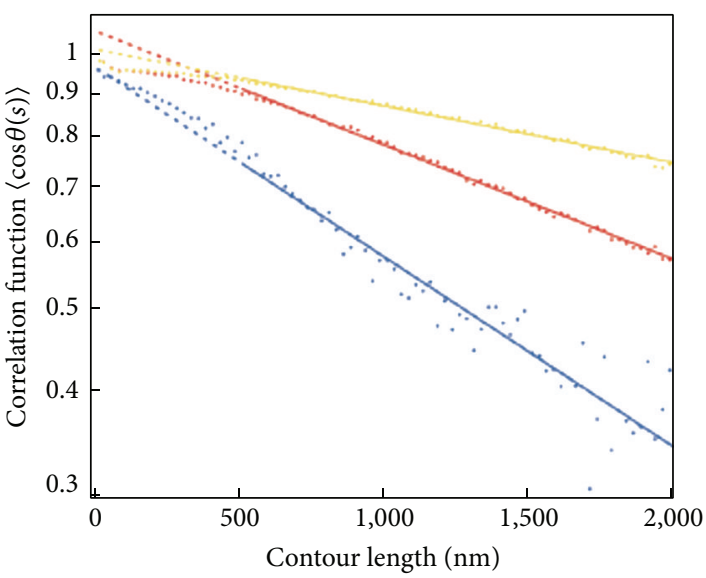

(b)

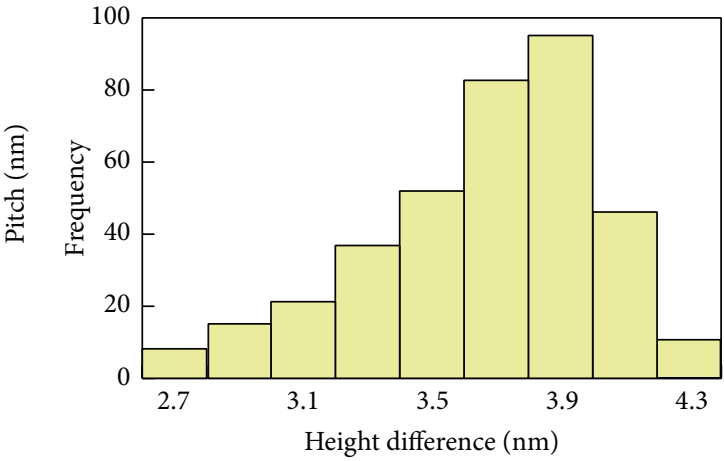

(d)
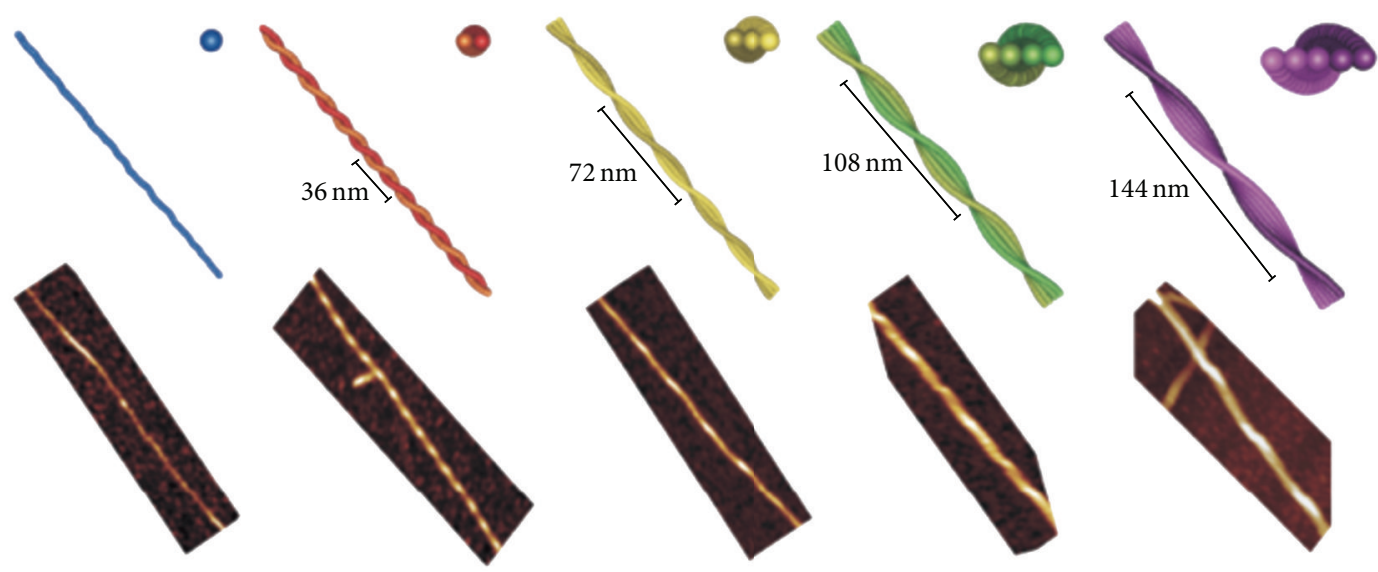

(e)

FIGURE 2: Nanomechanical properties of multistranded amyloid fibrils. (a) Schematic illustration of measuring the angle between two tangent vectors to extract the mechanical properties of the fibril using polymer chain statistics. (b) The ensemble averages of correlation functions (defined as cosine values of angles) for a multistranded fibril made of single (blue), two (red), and three (yellow) filaments, respectively, are measured from AFM experiments. The measured experimental results (indicated by blue, red, and yellow dots) are well fitted to theoretical predictions from polymer chain statistics (indicated by blue, red, and yellow lines). (c) Persistent length and helical pitch for amyloid fibrils composed of single, two, and three filaments, respectively. (d) The distribution of the difference between maximum and minimum AFM heights (thicknesses) of amyloid fibrils. (e) AFM images (shown in lower panel) and corresponding coarse-grained models (shown in upper panel) provide the left-handed multistranded amyloid fibrils made of one to five filaments, respectively. Figures are adopted with permission from [16]. 


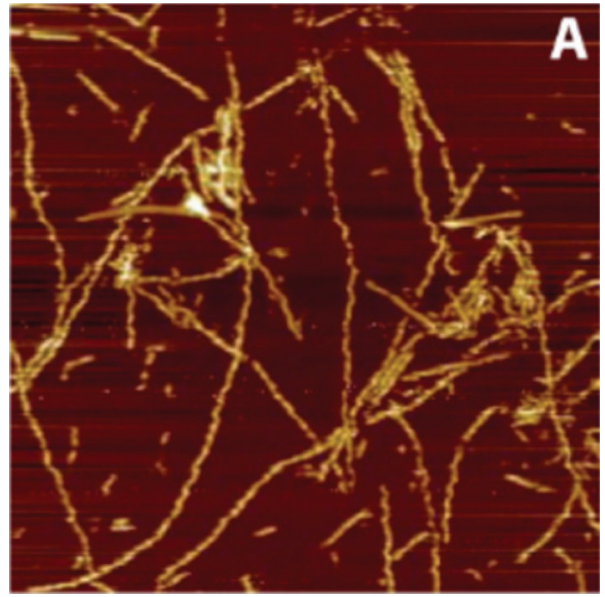

(a)

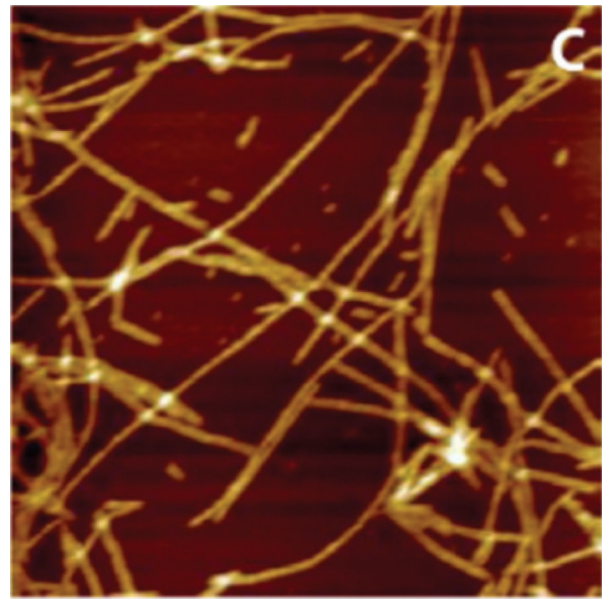

(c)
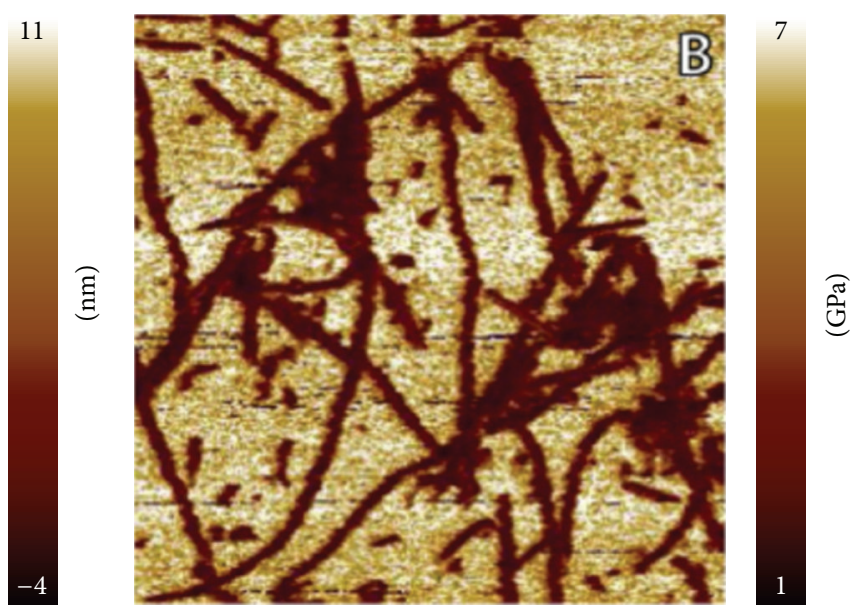

(b)
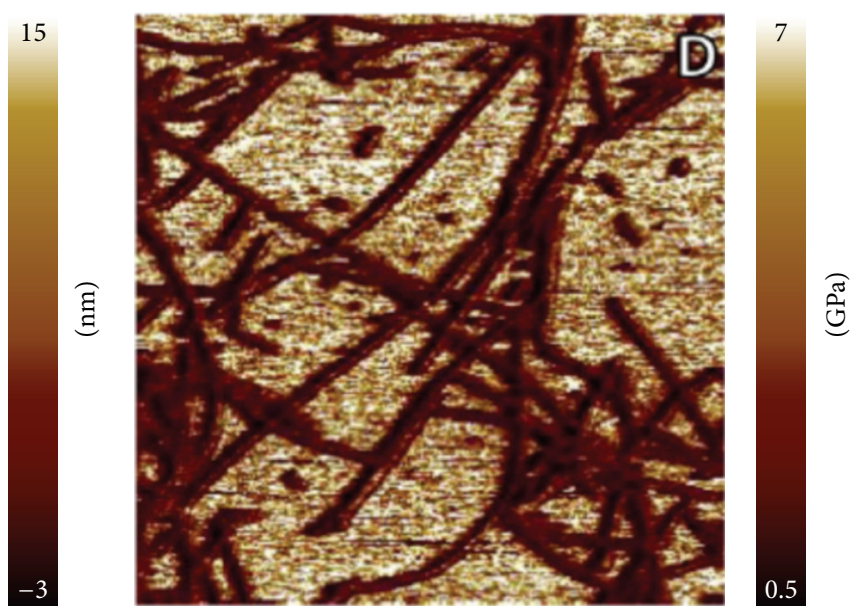

(d)

FIGURE 3: AFM peak force QNM- (quantitative nanomechanical mapping-) based experiments of $\alpha$-synuclein amyloid fibrils. (a) and (c) show the AFM topographic images of the fibrils, while (b) and (d) present the AFM peak force QNM images of the fibrils. Here, (a) and (b) are obtained from AFM QNM experiments of the fibrils in liquid environment, while (c) and (d) are acquired based on AFM QNM imaging of the fibrils in ambient condition. Figures are adopted from [20] under Creative Commons Attribution (CCA).

composed of single to five filaments. They found that the helical structure of a multistranded amyloid fibril is determined by interaction between filaments comprising the fibril. In addition, the persistent length of amyloid fibril is critically dependent on the number of filaments comprising the fibril such that the persistent length of the fibril increases with respect to the number of filaments. This has been elucidated based on helical ribbon model, which suggests that the increase of persistent length with respect to the number of filaments is attributed to the fact that the cross-sectional moment of inertia of the fibril increases with respect to the number of filaments. Furthermore, Usov and Mezzenga elaborated the theoretical model based on continuum elastic (beam) theory in order to understand the nanomechanical properties of amyloid fibrils with respect to their various conformations based on AFM experiments of the fibrils [73].

In addition to AFM imaging experiment, AFM-based nanoindentation has been considered to characterize the mechanical properties of amyloid fibrils. Adamcik and colleagues employed an AFM-based peak force QNM (quantitative nanomechanical mapping) technique to characterize the nanomechanical properties of $\beta$-lactoglobulin amyloid fibrils [19]. They found that the elastic modulus of $\beta$ lactoglobulin fibril is estimated as $3.3 \mathrm{GPa}$. A recent study by Sweers and coworkers [20] has suggested that the elastic modulus of $\alpha$-synuclein fibril was measured as $1.3 \sim 2.1 \mathrm{GPa}$ from AFM-based peak force QNM experiments (Figure 3). A previous study by Guo and Akhremitchev [21] reports the elastic modulus of insulin fibrils using AFM indentation experiments. Their study provides that the elastic modulus of insulin fibril is measured in a range of 5 to $50 \mathrm{MPa}$. In recent years, Gsponer and colleagues [28] utilized the AFM experiments (based on amplitude modulation-frequency modulation imaging, referred to as AM-FM imaging) to measure the mechanical properties of prion amyloid fibrils. They found that the radial elastic modulus of prion fibrils 


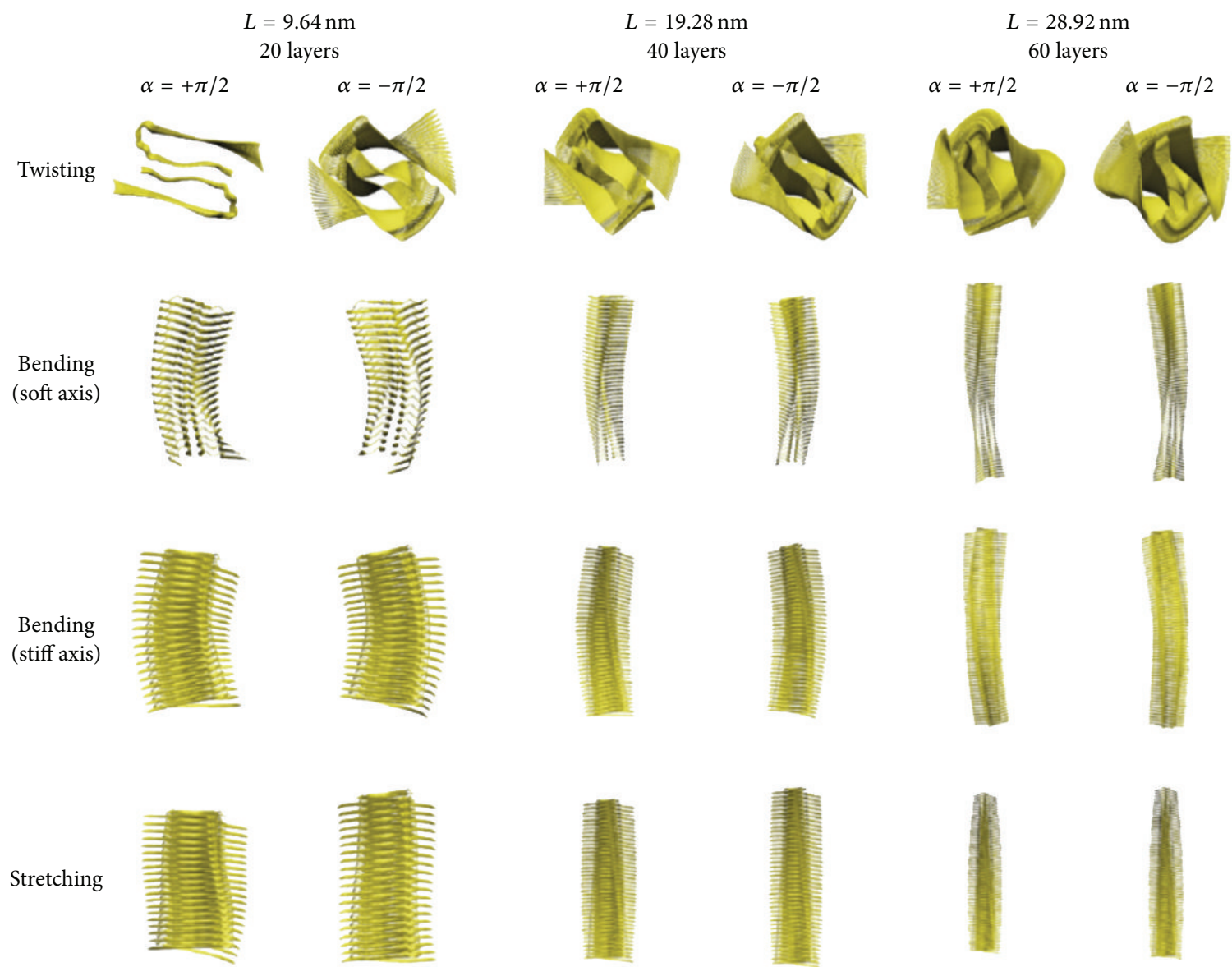

Figure 4: Vibrational characteristics of $\mathrm{A} \beta$ amyloid fibrils computed from elastic network model. Four low-frequency vibrational modes correspond to the twisting, two bending, and axial stretching modes, respectively. Here, low-frequency vibrational modes are shown for three different types of $\mathrm{A} \beta$ amyloid fibrils with their length of $9.64,19.28$, and $28.92 \mathrm{~nm}$, respectively. Figures are adopted with permission from [27].

is measured in a range of 0.5 to $1.3 \mathrm{GPa}$ and that this radial elastic property critically depends on their thickness in such a way that the radial elastic modulus of the fibrils decreases as their thickness increases.

\section{Computational Simulation-Based Mechanical Characterization of Amyloid Fibrils}

4.1. Nanomechanical Characterization Based on the Vibration of Amyloid Fibrils. A recent study by $\mathrm{Xu}$ and coworkers [27] considers ENM simulations to measure the vibrational characteristics of $\mathrm{A} \beta$ fibrils and their elastic properties. They found that the low-frequency dynamic modes of these fibrils correspond to twisting, bending, or axial stretching deformation modes (Figure 4) and that the elastic moduli of amyloid fibrils are dependent on their length scales. In addition, they report that the twisting (or helical) conformation of amyloid fibrils results in their isotropic bending property when the fibril length is $>150 \mathrm{~nm}$. Recently, our previous study [22] reports the nanomechanical properties of human islet amyloid polypeptide (hIAPP) fibrils, which are formed based on four different types of steric zipper patterns, based on ENM simulations. We found that as the fibril length increases, the torsional and axial stretching deformation modes of the fibril become the high-frequency dynamic modes, while the bending deformation mode corresponds to the low-frequency mode independent of fibril length (Figure 5(a)). This indicates that the thermal fluctuation behavior of an amyloid fibril is mostly contributed by the bending deformation mode, that is, a low-frequency mode. In addition, we have shown that the dependence of the bending rigidity (or persistent length) of amyloid fibrils on their length scales is well fitted to Timoshenko beam model (Figure 5(b)), which suggests that the bending property of the fibrils is attributed to competition between shear and bending deformation modes. Moreover, we have also studied the nanomechanical properties of prion amyloid fibrils based on ENM [30]. It is found that the mechanical properties of prion fibrils significantly depend on their $\beta$-helical structures and that the length-dependent mechanical properties of prion fibrils provide an insight into their critical size, at which prion infectivity is maximized. 


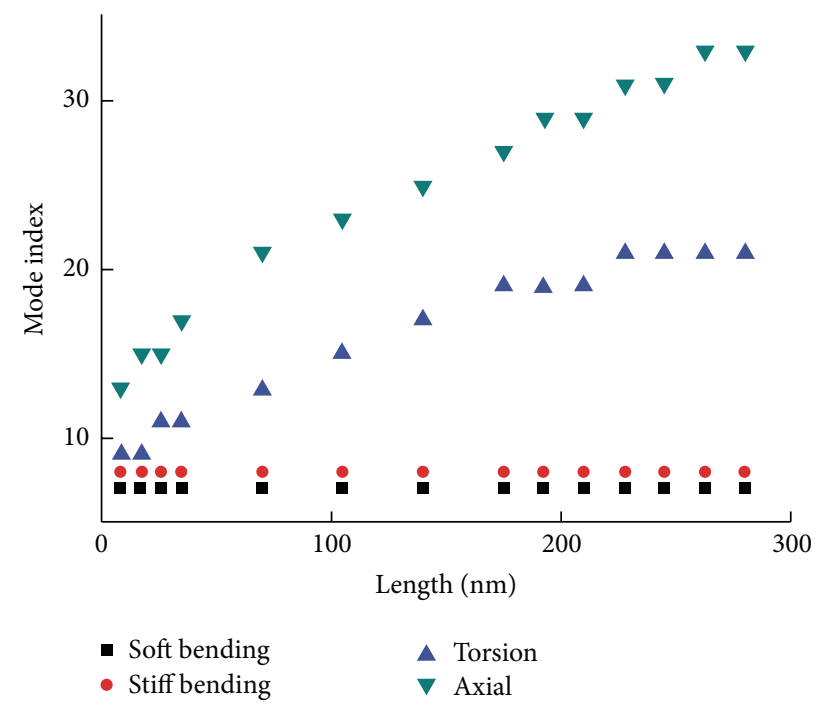

(a)

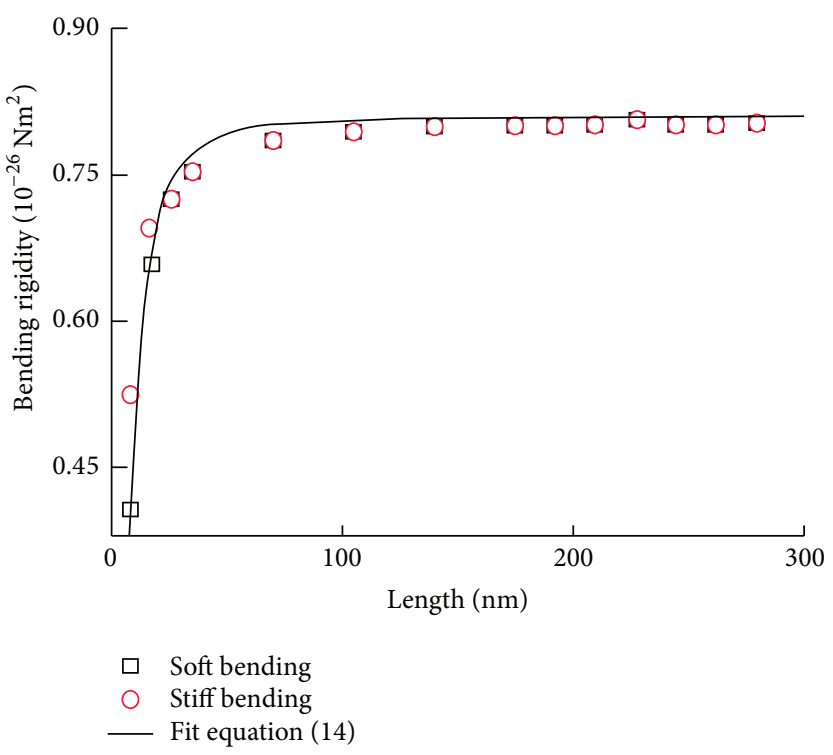

(b)

FIGURE 5: Length-dependent vibrational and nanomechanical properties of human islet amyloid polypeptide (hIAPP) fibrils computed from the elastic network model. (a) The mode index for three dominant deformation modes such as bending, torsional, and axial stretching deformation modes, respectively, is shown as a function of fibril length. As the fibril length increases, the torsional and axial deformation modes become the high-frequency vibrational modes, while the bending deformation modes are the low-frequency vibrational modes without any dependence on the fibril length. (b) The bending rigidity of hIAPP amyloid fibrils is shown as a function of their length scales based on ENM simulations. Figures are adopted with permission from [22].

In recent years, we have studied the vibrational characteristics of hIAPP (20-29) fibrils, which are formed based on the eight possible conformations of the fibrils as suggested in $[33,74]$ (Figure 6(a)), based on atomistic molecular dynamics (MD) simulations together with continuum elastic beam model [24]. We have shown that the fibril structure becomes stable when the fibril is formed based on antiparallel stacking of $\beta$-sheets (Figure 6(b)) and that the frequencies of the fibrils for their bending deformation modes are measured in the order of $0.2 \mathrm{THz}$, which is much larger than the frequencies measured by $4 \mathrm{D}$ electron microscopy experiments [36]. The discrepancy between frequencies measured from $\mathrm{MD}$ simulations and $4 \mathrm{D}$ electron microscopy experiments is attributed to the fact that the fibril length considered for MD simulation is less by a few orders of magnitude than that for 4D electron microscopy experiment. In addition, we have shown that the bending rigidity of hIAPP fibril becomes optimized when the fibril is formed based on the antiparallel stacking and that the mutation of phenylalanine to leucine in hIAPP (20-29) leads to the decrease of not only the structural stability but also the bending rigidity of hIAPP (20-29) fibril. Moreover, a recent study by Chang et al. [75] reports the effect of aromatic residue (i.e., phenylalanine) on the vibrational characteristics and mechanical properties of $\mathrm{A} \beta$ amyloid fibrils using MD simulations coupled with continuum elastic beam model. They found that aromatic residue such as phenylalanine plays a key role in stabilizing the structure of amyloid fibrils and in optimizing their mechanical properties.
Our recent study [34] reports the structure and vibrational (or equivalently mechanical) properties of a multistranded amyloid fibril by using atomistic MD simulations coupled with ENM. Specifically, MD simulation was used to understand the structure of a multistranded $\beta_{2}$ microglobulin fibril, whereas ENM was utilized to characterize their vibrational and mechanical properties (Figure $7(\mathrm{a})$ ). Our previous study found that the dependence of the helical structure of $\beta_{2}$-microglobulin fibril on the number of filaments comprising the fibril is attributed to the nonbonded interactions between filaments, which is consistent with AFM-based experimental study of $\beta$-lactoglobulin fibril [16]. In particular, MM-PBSA calculations suggest that as the number of filaments comprising the $\beta_{2}$-microglobulin fibril increases, the molecular mechanics energy of the fibril is decreased and it is mostly contributed by nonbonded energy such as electrostatic and van der Waals interaction energies [34]. Moreover, our previous study suggests the persistent length of amyloid fibrils as a function of the number of filaments comprising the fibril with using ENM simulation coupled with continuum beam model. The dependence of the persistent length on the number of filaments is well fitted to a scaling law of $L_{P} \propto\left[(n q)^{2}-\sin ^{2}(n q)\right]^{1 / 2}$ for a helical fibril model [73] (Figure 7(b)).

4.2. Mechanical Deformation Mechanisms of Amyloid Fibrils. Mechanical characterization of amyloid fibrils based on measuring their vibrational characteristics is insufficient to gain insight into the mechanical behaviors and properties 

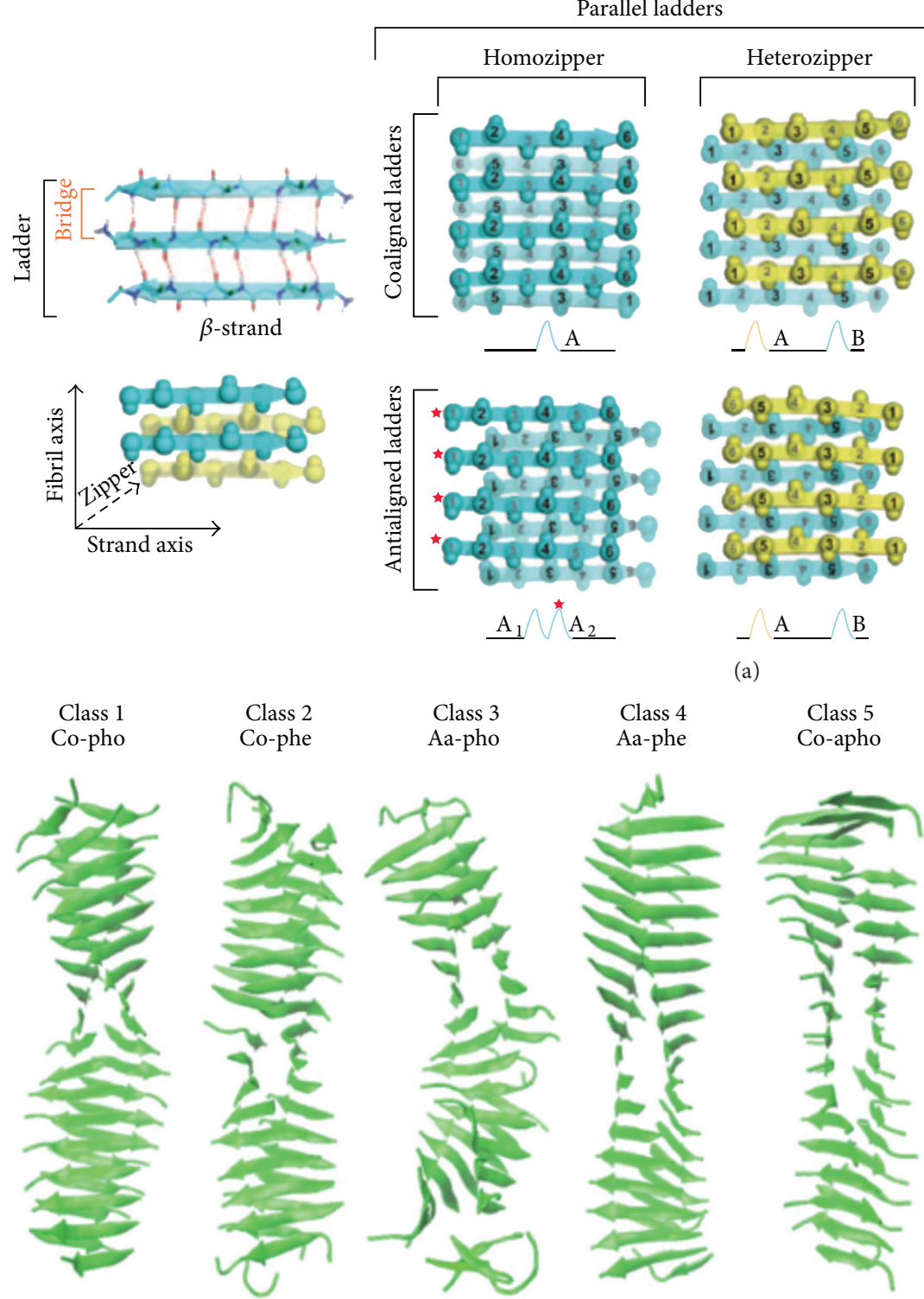

(a)
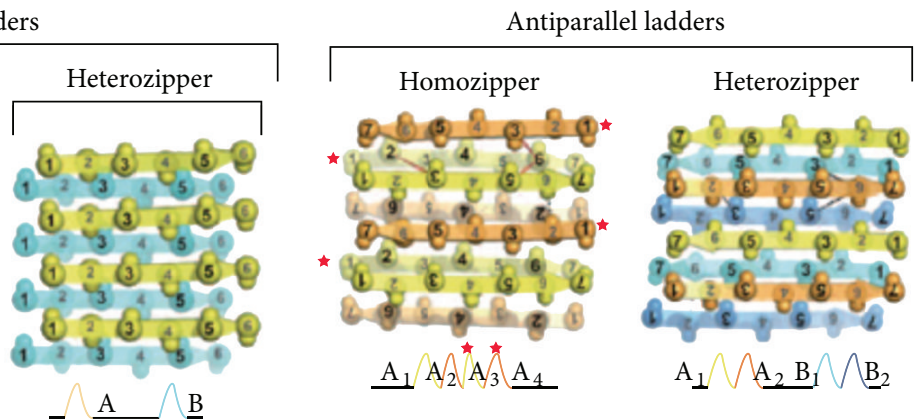

Coaligned ladders

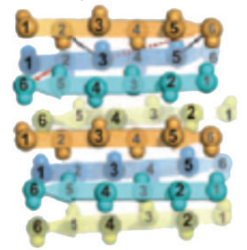

$\mathrm{A}_{1} \sim \mathrm{A}_{2} \mathrm{~B}_{1} \sim \underline{B}_{2}$
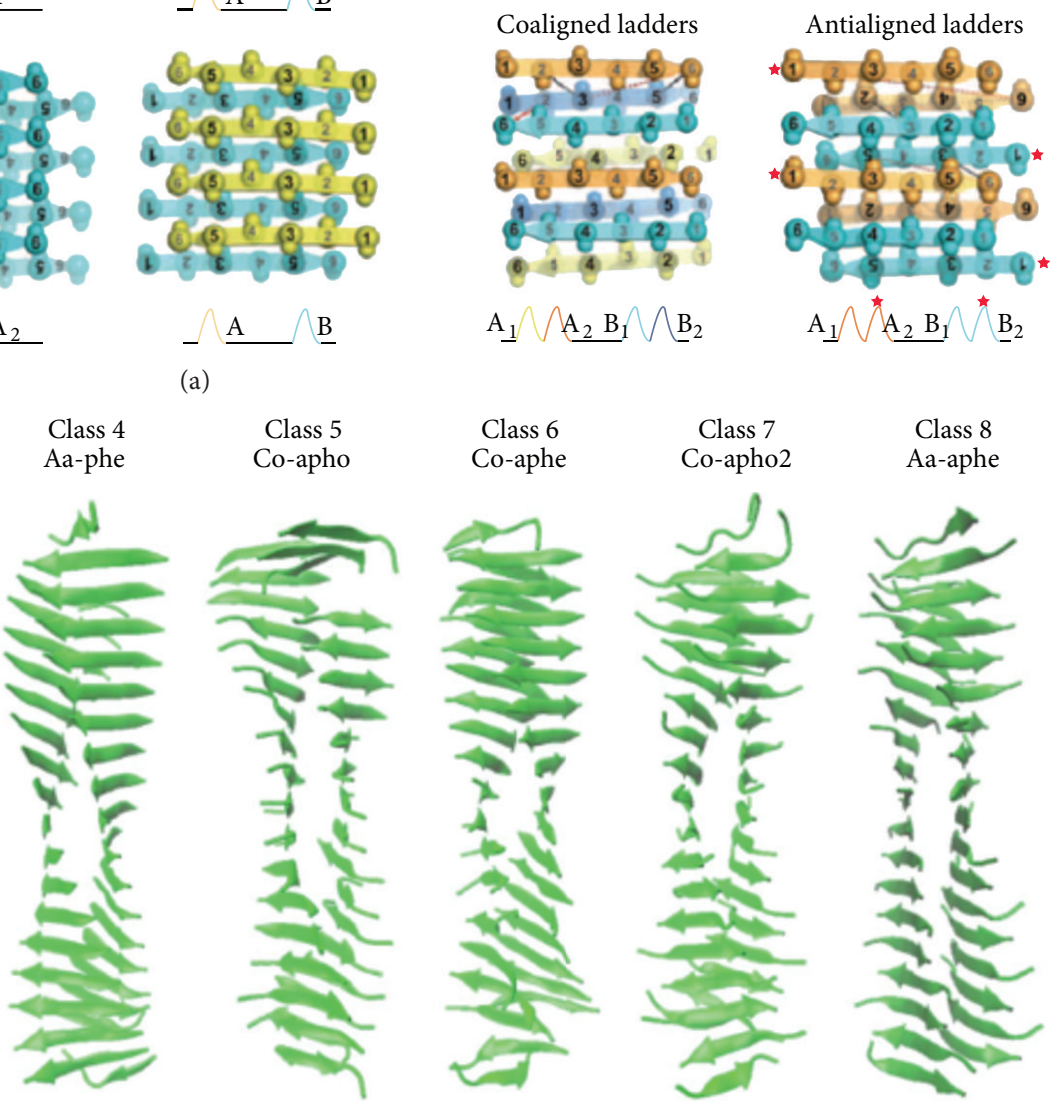

Class 6
Co-aphe

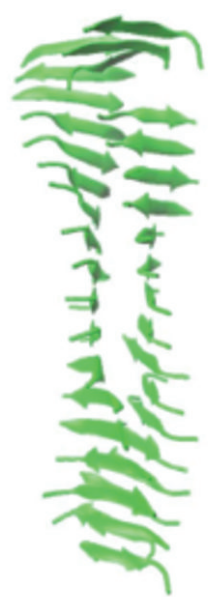

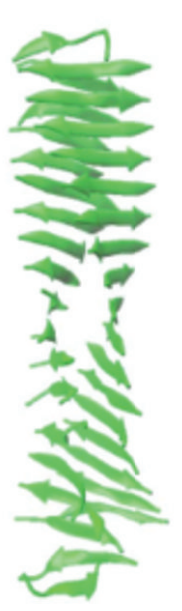

Class 8 Aa-aphe

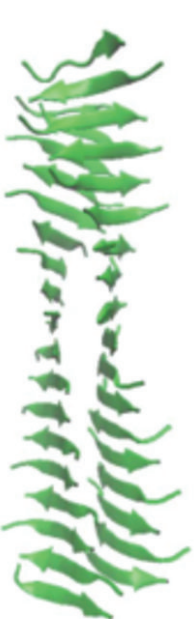

(b)

FIGURE 6: (a) Structural models of amyloid fibrils. These structural models are theoretically provided based on $\beta$-sheet stacking patterns such as parallel or antiparallel stacking. Figures are adopted with permission from [33]. (b) Atomistic molecular dynamics simulations provide the equilibrium structures of hIAPP amyloid fibrils based on the structural models. Figures are adopted from [24] under Creative Commons Attribution (CCA).

of amyloid fibrils, as the vibrational properties of the fibrils are correlated with only their elastic moduli [76]. In particular, the vibrational characteristics of amyloid fibrils cannot provide the quantitative insight into the fracture behaviors and properties of amyloid fibrils. Here, we take a look at the mechanical deformation mechanisms and properties of amyloid fibrils based on two types of deformation modes such as (i) axial deformation and (ii) bending-like deformation.

First, we consider recent attempts to characterize the axial deformation behavior of amyloid fibrils. A recent study by Dong et al. [35] reports the axial extension of prion fibrils using optical tweezer force spectroscopy. In their experiment [35], it is found that the prion fibrils can resist force up to $250 \mathrm{pN}$ and that discontinuities in the force-extension curve (before the fibril is ruptured) are observed possibly due to the partial unfolding of prion proteins comprising the fibril (Figure 8). SMD simulation-based study by Lee et al. [77] provides that the mechanical behavior of polymorphic amyloid fibrils is critically dependent on their molecular structures. In particular, an amyloid fibril formed based on antiparallel stacking of $\beta$-sheets can sustain a force even up to $\sim 1500 \mathrm{pN}$. This value of the force is larger than a force that other fibrils, which are formed based on other stacking 


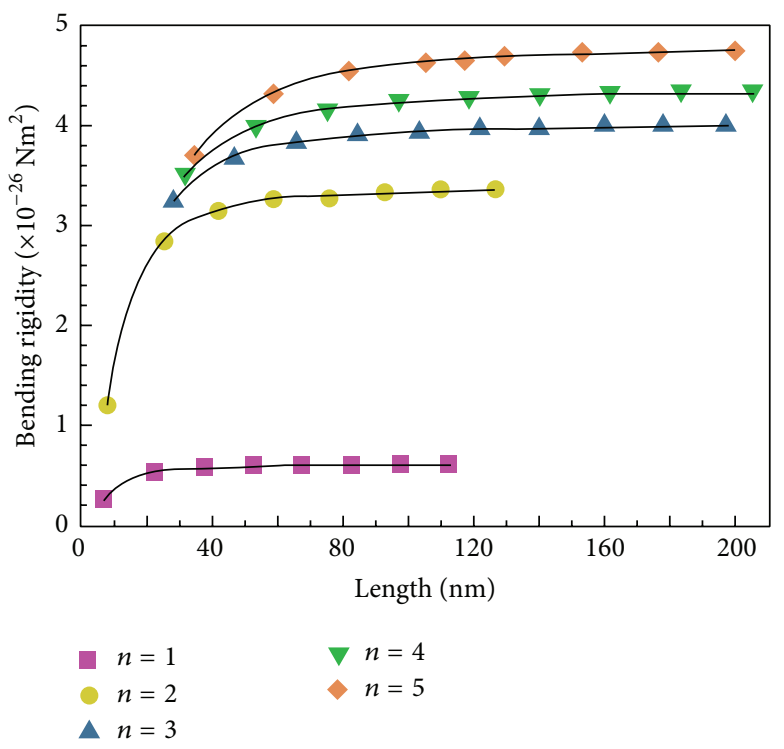

(a)

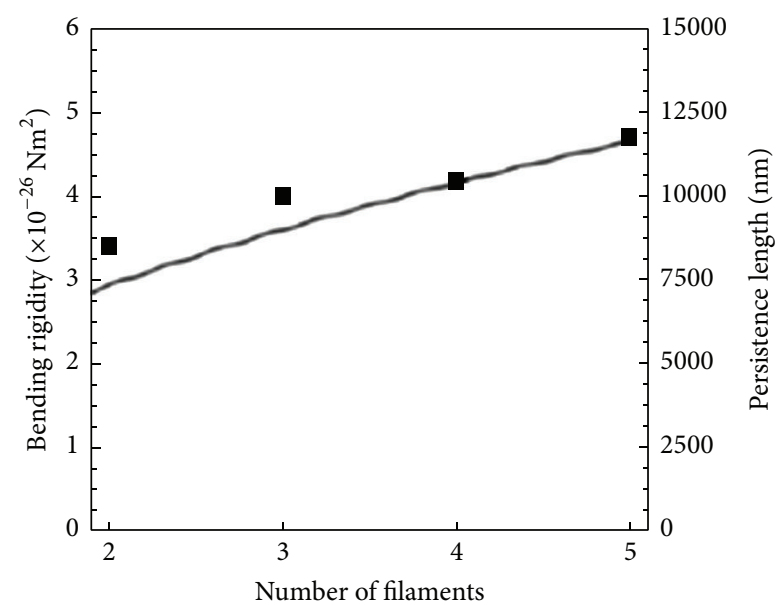

(b)

Figure 7: Nanomechanical properties of $\beta_{2}$-microglobulin fibrils made of one to five filaments, respectively. (a) The length-dependent effective bending rigidity of $\beta_{2}$-microglobulin fibrils composed of one to five filaments, respectively. (b) The intrinsic bending rigidity of $\beta_{2}$-microglobulin fibrils made of two to five filaments, respectively, is shown as a function of the number of filaments comprising the fibrils. The dots indicate the results of ENM simulations, while solid line presents the theoretical prediction from continuum helical fibril model. Figures are adopted with permission from [34].
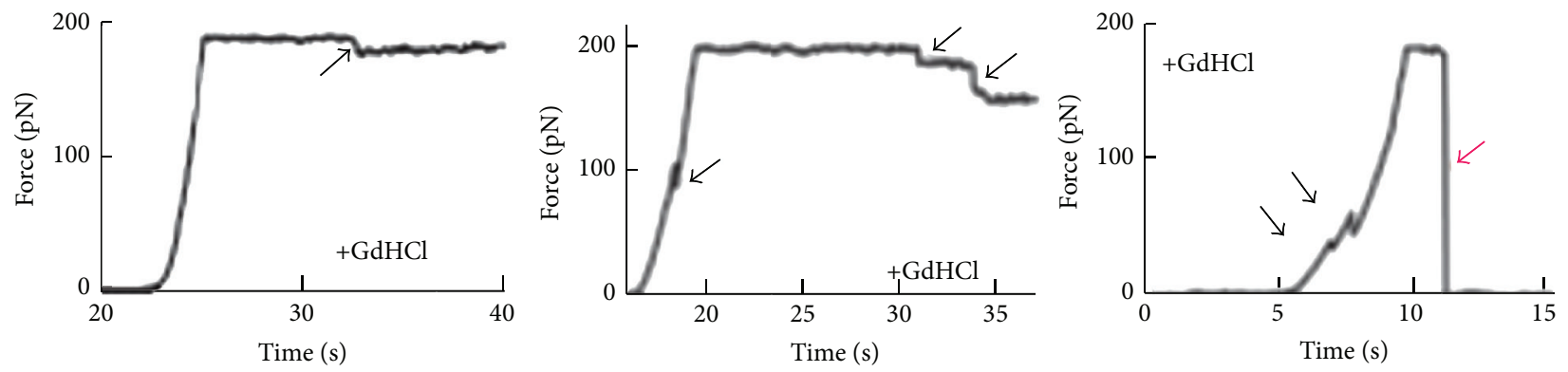

(a)

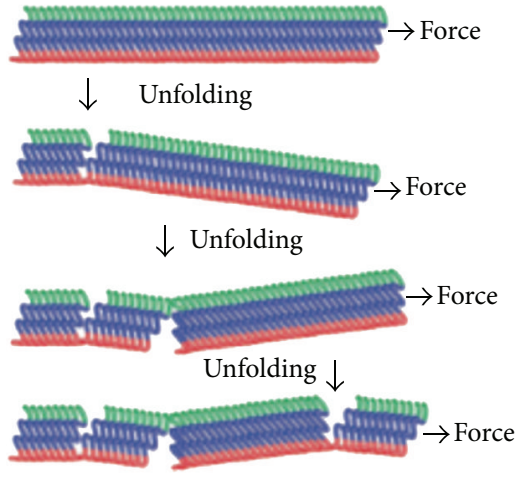

(b)

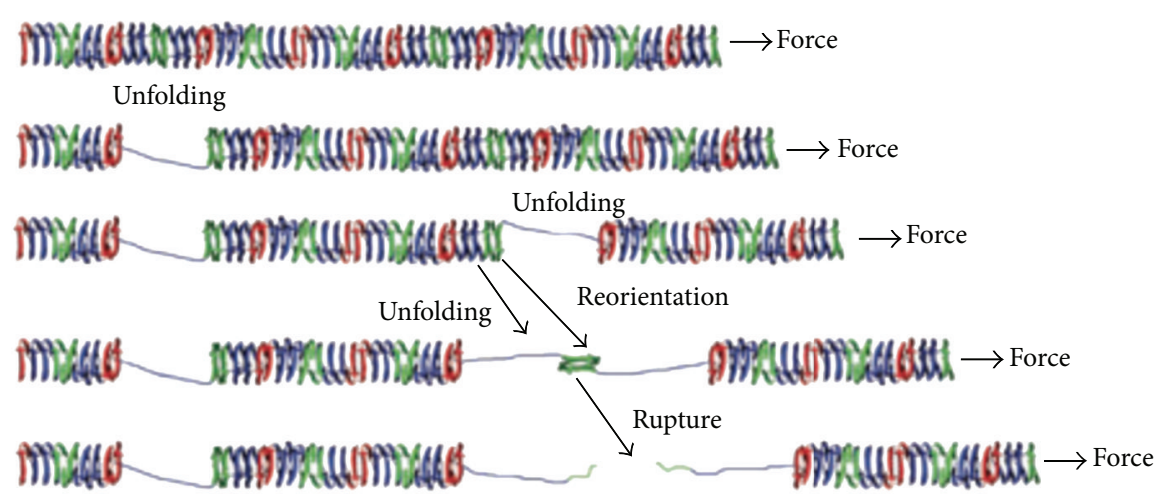

(c)

FIGURE 8: Optical tweezer-based stretching experiment of prion fibrils. (a) Three representative force curves for prion fibril when it is extended with a constant pulling speed. Arrows indicate discontinuities in a force-extension curve due to partial unfolding of prion proteins. (b) Fracture mechanism of amyloid fibril made of $\beta$-helices due to partial unfolding. (c) Fracture mechanism of amyloid fibril made of $\beta$-sheets due to partial unfolding. Figures are adopted with permission from [35]. 


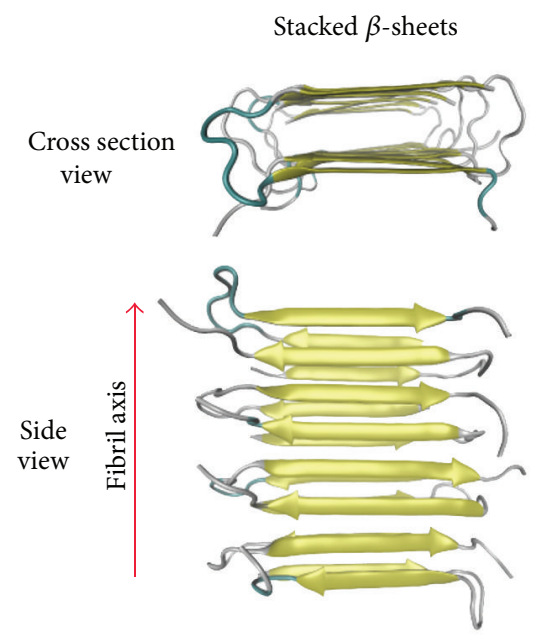

(a)

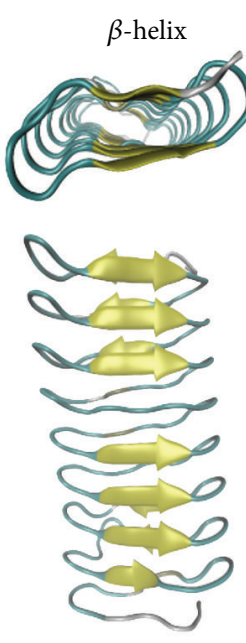

(b)
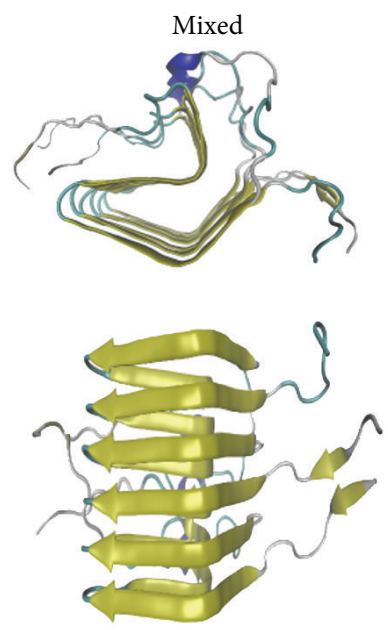

(c)

Figure 9: Three different structural types of amyloid fibrils. Amyloid fibrils can be formed based on (a) stacked $\beta$-sheets, (b) $\beta$-helices, or (c) stacked $\beta$-helices. Figures are redrawn using VMD package for these three types of structures, whose information is provided in the protein data bank (PDB) with PDB codes of 2LNQ, 1P9H, and 2KJ3, respectively.

patterns, can resist. In recent years, Solar and Buehler [29] report the tensile deformation and failure of amyloid fibrils using SMD simulations. They considered three types of amyloid fibrils based on their structural features (Figure 9): (a) a fibril formed based on stacking of $\beta$-sheets, (b) a fibril constructed based on $\beta$-helix, and (c) a fibril established based on mixed structure, that is, stacking of $\beta$-helices. The mechanical strength of an amyloid fibril can be maximized (i.e., up to $1000 \mathrm{MPa}$ ) when it is formed based on a mixed structure. By contrast, the strength is estimated as 200 $400 \mathrm{MPa}$ for a fibril formed by stacking of $\beta$-sheets, and it is measured as $\sim 600 \mathrm{MPa}$ for the fibril constructed based on $\beta$-helix.

Now, we take into account recent efforts to characterize the bending deformation of an amyloid fibril. A previous study by Smith et al. [26] reports the three-point bendinglike deformation of an amyloid fibril using atomic force microscopy (AFM) experiment. It is found that the insulin amyloid fibril exhibits the elastic modulus of $\sim 3.3 \mathrm{GPa}$, mechanical strength of $\sim 600 \mathrm{MPa}$, and bending rigidity of $\sim 9.1 \times 10^{-26} \mathrm{~N} \cdot \mathrm{m}^{2}$, respectively. Our previous study [23] considers the bending deformation mechanisms of amyloid fibrils using SMD simulations. In our previous study, it was found that the deformation mechanism of amyloid fibrils is significantly dependent on their length scales (Figure 10(a)). In particular, when a short fibril is deformed, it undergoes shear-like deformation. By contrast, the bending deformation occurs when a long fibril is deformed. Furthermore, it was shown that Timoshenko beam model is able to capture the length-dependent stiffness of amyloid fibrils (Figure 10(b)) and that their length-dependent fracture behavior (Figure 10(c)) is attributed to the length-dependent mechanisms of hydrogen bond ruptures [23]. In addition, our recent study [78] has shown the effect of boundary conditions in the fracture behavior of amyloid fibrils. Moreover, a recent study by Kim et al. [79] provides the anisotropic bending deformation mechanisms of hIAPP amyloid fibrils using atomistic SMD simulations. Their study has shown that the fracture property (e.g., rupture force) of amyloid fibril is critically dependent on bending direction, which highlights the important role of loading mode in the fracture properties of amyloid fibrils.

\section{Discussion}

In this review article, we address recent attempts to characterize the nanomechanical properties of amyloid fibrils measured from computational simulations and/or AFM experiments together with theoretical models. The nanomechanical properties of amyloid fibrils are summarized in Table 1, which shows that the elastic modulus of amyloid fibrils is evaluated in the order of 1 to $10 \mathrm{GPa}$ and that their mechanical strength is measured in the order of $1 \mathrm{GPa}$. It should be noted that though both amyloid fibril and spider silk crystal are made of stacked $\beta$-sheets, the mechanical strength of amyloid fibril is less than that of a spider silk crystal, which is attributed to the different orientation of stacked $\beta$-sheets with respect to the fibril axis [31].

Here, we note that most of recent works reviewed in this work do not consider the effect of physiological conditions in the nanomechanical properties of amyloid fibrils. It has recently been found that physiological conditions play a crucial role in the formation and structure of amyloid proteins. For instance, a recent study by Lee et al. [80] reports a critical role that the physiological condition such as the $\mathrm{pH}$ of a solvent plays in the morphology of amyloid fibrils. In addition, Mizuno and coworkers [81] found the dependence of HET-s prion amyloid fibril structure on the $\mathrm{pH}$ of a solvent. Moreover, it has been debated whether metal ion promotes the formation of amyloid oligomers or fibrils, as previous 

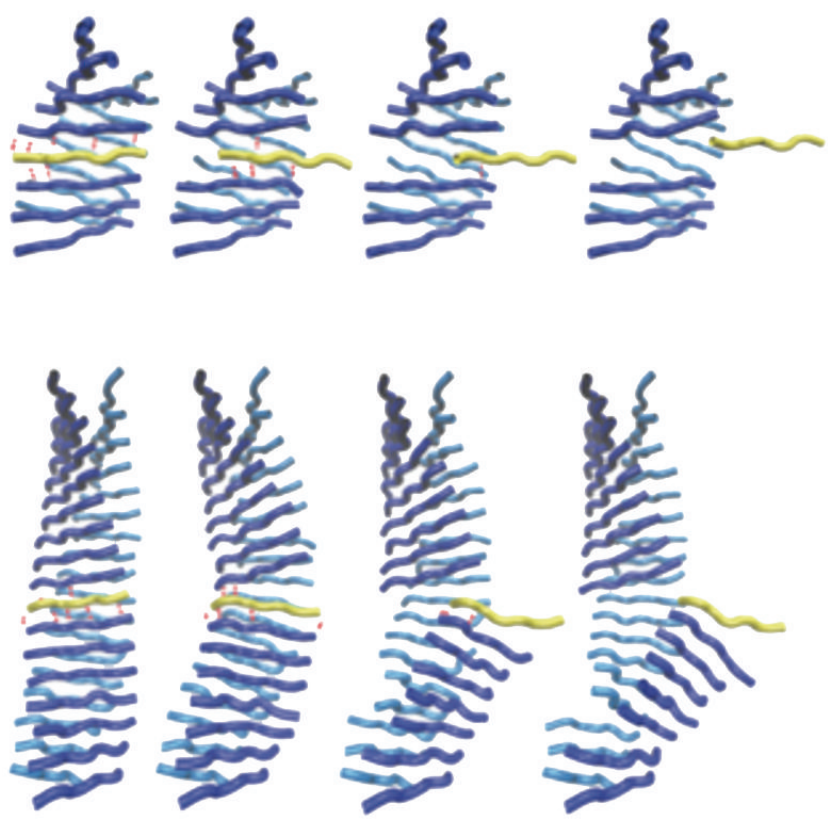

(a)

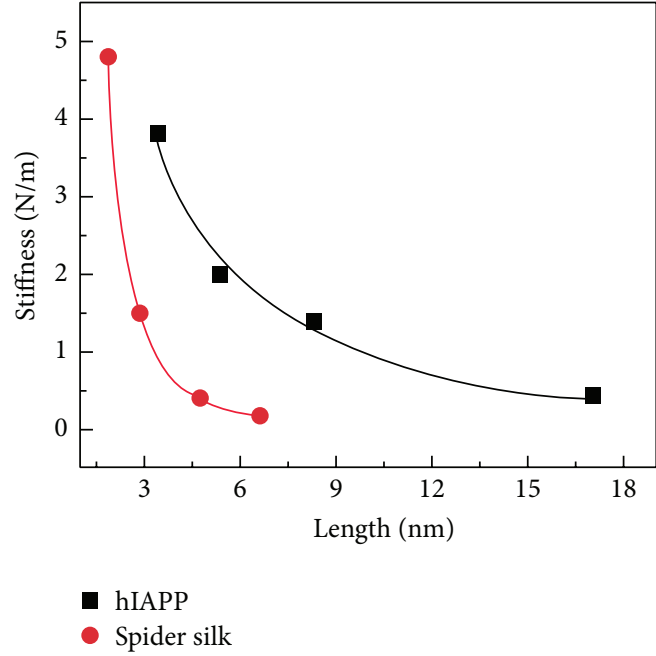

(b)

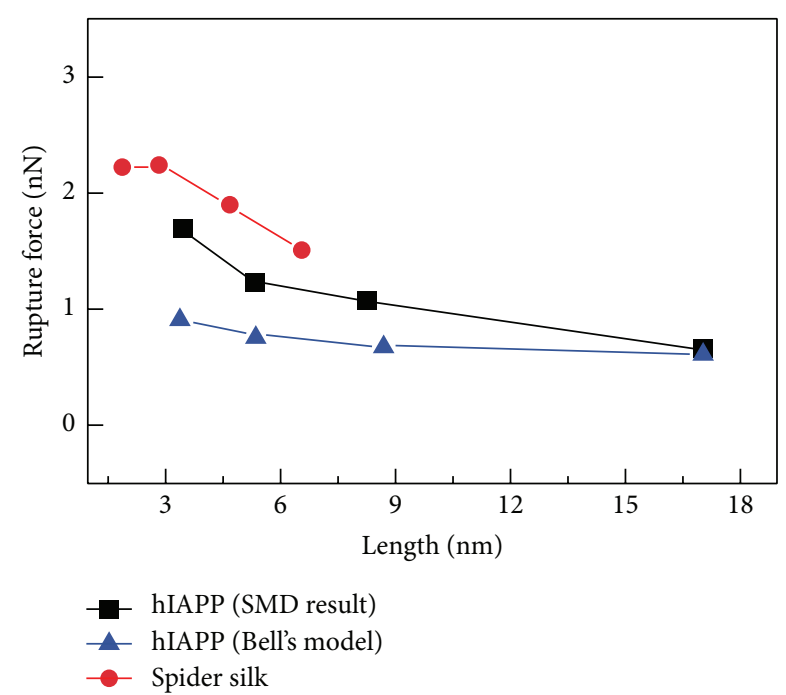

(c)

FIGURE 10: (a) Length-dependent mechanical deformation mechanisms of amyloid fibrils. The upper panel shows the shear-like deformation of a short fibril, while the lower panel provides the bending deformation of a long fibril. (b) Length-dependent elastic stiffness of amyloid fibrils (black) and spider silk crystals (red). (c) Length-dependent rupture forces of amyloid fibrils (black) and spider silk crystals (red). Figures are adopted with permission from [23].

studies [82-84] report that the high concentration of metal ions was found for patients suffering from neurodegenerative diseases. In addition, recent studies $[85,86]$ have shown the important role of metal ion in the formation and structure of amyloid fibrils. Despite these recent studies showing the role of physiological condition in the formation and structure of amyloid proteins, how physiological condition may make an impact on the nanomechanical properties of amyloid fibrils has remained elusive. Future studies may be directed towards understanding how physiological conditions affect the nanomechanical properties of amyloid fibrils.

In conclusion, this review article summarizes the excellent nanomechanical properties of amyloid fibrils, which are measured with using experiments (based on AFM) and computational simulations (based on atomistic simulations or ENM). The nanomechanical characterization of amyloid fibrils may allow for further insight into not only the mechanics-driven biological functions of the fibrils but also the design principles showing how the 
TABLE 1: Nanomechanical properties of amyloid fibrils measured from simulations and experiments.

\begin{tabular}{|c|c|c|c|c|c|c|c|}
\hline Material & Measurement method & Length (nm) & $\begin{array}{l}\text { Bending rigidity } \\
\left(\times 10^{-26} \mathrm{~N} \cdot \mathrm{m}^{2}\right)\end{array}$ & $\begin{array}{c}\text { Young's } \\
\text { modulus (GPa) }\end{array}$ & $\begin{array}{l}\text { Shear modulus } \\
(\mathrm{GPa})\end{array}$ & $\begin{array}{l}\text { Strength } \\
(\mathrm{GPa})\end{array}$ & Ref. \\
\hline hIAPP $_{20-29}$ fibril & $\begin{array}{l}\text { Steered molecular dynamics } \\
\text { (simulation) }\end{array}$ & $3.41-17.5$ & $7.73-37.7$ & & $5.97-6.71$ & $4-8$ & [23] \\
\hline hIAPP $_{20-29}$ fibril & $\begin{array}{l}\text { Elastic network model } \\
\text { (simulation) }\end{array}$ & $10-300$ & $\sim 8$ & $12-14$ & 1.1 & & {$[22]$} \\
\hline hIAPP $_{20-29}$ fibril & $\begin{array}{l}\text { Molecular dynamics } \\
\text { (simulation) }\end{array}$ & 10 & $0.01-0.04$ & $0.4-0.6$ & & & {$[24]$} \\
\hline $\mathrm{A} \beta_{1-40}$ fibril & $\begin{array}{l}\text { Cryoelectron microscopy } \\
\text { (experiment) }\end{array}$ & $500-1000$ & $\sim 13$ & & 0.0127 & & [17] \\
\hline $\mathrm{A} \beta_{1-40}$ fibril & $\begin{array}{l}\text { Elastic network model } \\
\text { (simulation) }\end{array}$ & $\sim 30$ & $21-63$ & & $4.3-5.6$ & & [27] \\
\hline $\begin{array}{l}\beta \text {-lactoglobulin } \\
\text { fibril }\end{array}$ & AFM imaging experiment & $500-15,000$ & $0.4-1.6$ & $\sim 4$ & & & {$[16]$} \\
\hline $\begin{array}{l}\beta \text {-lactoglobulin } \\
\text { fibril }\end{array}$ & $\begin{array}{l}\text { AFM indentation experiment } \\
\text { (peak force QNM) }\end{array}$ & $500-15,000$ & & 3.3 & & & {$[19]$} \\
\hline Mouse prion fibril & $\begin{array}{l}\text { AFM experiments (AM-FM } \\
\text { imaging) }\end{array}$ & $>1000$ & & $0.5-1.36$ & & & {$[28]$} \\
\hline$\alpha$-synuclein fibril & $\begin{array}{l}\text { AFM indentation (peak force } \\
\text { QNM) }\end{array}$ & $>1000$ & & $1.2 \pm 0.2$ & & & [20] \\
\hline Insulin fibril & AFM bending experiment & $>\sim 1500$ & $\sim 9.1$ & & $\sim 0.28$ & & [26] \\
\hline Insulin fibril & AFM imaging experiment & $>\sim 2000$ & $\sim 17$ & & $\sim 0.13$ & & [26] \\
\hline HET-s prion fibril & $\begin{array}{l}\text { Steered molecular dynamics } \\
\text { (simulation) }\end{array}$ & 5.38 & & 9.8 & & 0.917 & [29] \\
\hline HET-s prion fibril & $\begin{array}{l}\text { Elastic network model } \\
\text { (simulation) }\end{array}$ & 8.93 & 0.115 & 1.5 & & & [30] \\
\hline Spider silk crystal & $\begin{array}{l}\text { Steered molecular dynamics } \\
\text { (simulation) }\end{array}$ & $2-7$ & $\sim 2.8$ & & 4.6 & & {$[12]$} \\
\hline Spider silk crystal & $\begin{array}{l}\text { Steered molecular dynamics } \\
\text { (simulation) }\end{array}$ & & & $30-70$ & & & {$[31]$} \\
\hline
\end{tabular}

properties of protein materials can be determined or controlled.

\section{Disclosure}

The funder had no role in publishing this paper.

\section{Competing Interests}

The authors declare there are no competing interests regarding the publication of this paper.

\section{Authors' Contributions}

Bumjoon Choi and Taehee Kim made equal contribution to this work.

\section{Acknowledgments}

This work was supported by the National Research Foundation of Korea (NRF), under Grant no. NRF2015R1A2A2A04002453, and Korea Institute of Science and
Technology Information (KISTI) under Grant no. KSC2015-C3-051 (to Kilho Eom) and NRF under Grant no. NRF-2013R1A1A2053613 (to Sang Woo Lee).

\section{References}

[1] I. Cherny and E. Gazit, "Amyloids: not only pathological agents but also ordered nanomaterials," Angewandte ChemieInternational Edition, vol. 47, no. 22, pp. 4062-4069, 2008.

[2] B. H. Toyama and J. S. Weissman, "Amyloid structure: conformational diversity and consequences," Annual Review of Biochemistry, vol. 80, pp. 557-585, 2011.

[3] J. E. Straub and D. Thirumalai, "Toward a molecular theory of early and late events in monomer to amyloid fibril formation," Annual Review of Physical Chemistry, vol. 62, pp. 437-463, 2011.

[4] G. Lee, W. Lee, H. Lee, C. Y. Lee, K. Eom, and T. Kwon, "Selfassembled amyloid fibrils with controllable conformational heterogeneity," Scientific Reports, vol. 5, Article ID 16220, 2015.

[5] M. B. Pepys, "Amyloidosis," Annual Review of Medicine, vol. 57, pp. 223-241, 2006.

[6] F. Chiti and C. M. Dobson, "Protein misfolding, functional amyloid, and human disease," Annual Review of Biochemistry, vol. 75, pp. 333-366, 2006. 
[7] G. Merlini and V. Bellotti, "Molecular mechanisms of amyloidosis," The New England Journal of Medicine, vol. 349, no. 6, pp. 583-596, 2003.

[8] J. W. M. Höppener, B. Ahrén, and C. J. M. Lips, "Islet amyloid and type 2 diabetes mellitus," The New England Journal of Medicine, vol. 343, no. 6, pp. 411-419, 2000.

[9] C. Haass and D. J. Selkoe, "Soluble protein oligomers in neurodegeneration: lessons from the Alzheimer's amyloid $\beta$ peptide," Nature Reviews Molecular Cell Biology, vol. 8, no. 2, pp. 101-112, 2007.

[10] T. P. J. Knowles and M. J. Buehler, "Nanomechanics of functional and pathological amyloid materials," Nature Nanotechnology, vol. 6, no. 8, pp. 469-479, 2011.

[11] C. Rapezzi, C. C. Quarta, L. Riva et al., "Transthyretin-related amyloidoses and the heart: a clinical overview," Nature Reviews Cardiology, vol. 7, no. 7, pp. 398-408, 2010.

[12] S. Keten, Z. Xu, B. Ihle, and M. J. Buehler, "Nanoconfinement controls stiffness, strength and mechanical toughness of B-sheet crystals in silk," Nature Materials, vol. 9, no. 4, pp. 359-367, 2010.

[13] K. Eom, P.-C. Li, D. E. Makarov, and G. J. Rodin, "Relationship between the mechanical properties and topology of cross-linked polymer molecules: parallel strands maximize the strength of model polymers and protein domains," The Journal of Physical Chemistry B, vol. 107, no. 34, pp. 8730-8733, 2003.

[14] C. C. Vandenakker, M. F. M. Engel, K. P. Velikov, M. Bonn, and G. H. Koenderink, "Morphology and persistence length of amyloid fibrils are correlated to peptide molecular structure," Journal of the American Chemical Society, vol. 133, no. 45, pp. 18030-18033, 2011.

[15] F. S. Ruggeri, J. Adamcik, J. S. Jeong, H. A. Lashuel, R. Mezzenga, and G. Dietler, "Influence of the $\beta$-sheet content on the mechanical properties of aggregates during amyloid fibrillization," Angewandte Chemie-International Edition, vol. 54, no. 8, pp. 2462-2466, 2015.

[16] J. Adamcik, J.-M. Jung, J. Flakowski, P. De Los Rios, G. Dietler, and R. Mezzenga, "Understanding amyloid aggregation by statistical analysis of atomic force microscopy images," Nature Nanotechnology, vol. 5, no. 6, pp. 423-428, 2010.

[17] C. Sachse, N. Grigorieff, and M. Fändrich, "Nanoscale flexibility parameters of Alzheimer amyloid fibrils determined by electron cryo-microscopy," Angewandte Chemie-International Edition, vol. 49, no. 7, pp. 1321-1323, 2010.

[18] I. Usov and R. Mezzenga, "FiberApp: an open-source software for tracking and analyzing polymers, filaments, biomacromolecules, and fibrous objects," Macromolecules, vol. 48, no. 5, pp. 1269-1280, 2015.

[19] J. Adamcik, A. Berquand, and R. Mezzenga, "Single-step direct measurement of amyloid fibrils stiffness by peak force quantitative nanomechanical atomic force microscopy," Applied Physics Letters, vol. 98, no. 19, Article ID 193701, 2011.

[20] K. Sweers, K. van der Werf, M. Bennink, and V. Subramaniam, "Nanomechanical properties of $\alpha$-synuclein amyloid fibrils: a comparative study by nanoindentation, harmonic force microscopy, and Peakforce QNM," Nanoscale Research Letters, vol. 6, no. 1, article 270, 2011.

[21] S. Guo and B. B. Akhremitchev, "Packing density and structural heterogeneity of insulin amyloid fibrils measured by AFM nanoindentation," Biomacromolecules, vol. 7, no. 5, pp. 16301636, 2006.

[22] G. Yoon, J. Kwak, J. I. Kim, S. Na, and K. Eom, "Mechanical characterization of amyloid fibrils using coarse-grained normal mode analysis," Advanced Functional Materials, vol. 21, no. 18, pp. 3454-3463, 2011.

[23] B. Choi, G. Yoon, S. W. Lee, and K. Eom, "Mechanical deformation mechanisms and properties of amyloid fibrils," Physical Chemistry Chemical Physics, vol. 17, no. 2, pp. 1379-1389, 2015.

[24] G. Yoon, M. Lee, J. I. Kim, S. Na, and K. Eom, "Role of sequence and structural polymorphism on the mechanical properties of amyloid fibrils," PLoS ONE, vol. 9, no. 2, article e88502, 2014.

[25] J. M. Gosline, P. A. Guerette, C. S. Ortlepp, and K. N. Savage, "The mechanical design of spider silks: from fibroin sequence to mechanical function," Journal of Experimental Biology, vol. 202, no. 23, pp. 3295-3303, 1999.

[26] J. F. Smith, T. P. J. Knowles, C. M. Dobson, C. E. MacPhee, and M. E. Welland, "Characterization of the nanoscale properties of individual amyloid fibrils," Proceedings of the National Academy of Sciences of the United States of America, vol. 103, no. 43, pp. 15806-15811, 2006.

[27] Z. Xu, R. Paparcone, and M. J. Buehler, "Alzheimer's A $\beta(1-40)$ amyloid fibrils feature size-dependent mechanical properties," Biophysical Journal, vol. 98, no. 10, pp. 2053-2062, 2010.

[28] G. Lamour, C. K. Yip, H. Li, and J. Gsponer, "High intrinsic mechanical flexibility of mouse prion nanofibrils revealed by measurements of axial and radial young's moduli," ACS Nano, vol. 8, no. 4, pp. 3851-3861, 2014.

[29] M. Solar and M. J. Buehler, "Tensile deformation and failure of amyloid and amyloid-like protein fibrils," Nanotechnology, vol. 25, no. 10, Article ID 105703, 2014.

[30] G. Yoon, Y. K. Kim, K. Eom, and S. Na, "Relationship between disease-specific structures of amyloid fibrils and their mechanical properties," Applied Physics Letters, vol. 102, no. 1, Article ID 011914, 2013.

[31] S. Xiao, S. Xiao, and F. Gräter, "Dissecting the structural determinants for the difference in mechanical stability of silk and amyloid beta-sheet stacks," Physical Chemistry Chemical Physics, vol. 15, no. 22, pp. 8765-8771, 2013.

[32] T. P. Knowles, A. W. Fitzpatrick, S. Meehan et al., "Role of intermolecular forces in defining material properties of protein nanofibrils," Science, vol. 318, no. 5858, pp. 1900-1903, 2007.

[33] J. T. Nielsen, M. Bjerring, M. D. Jeppesen et al., "Unique identification of supramolecular structures in amyloid fibrils by solidstate NMR spectroscopy," Angewandte Chemie-International Edition, vol. 48, no. 12, pp. 2118-2121, 2009.

[34] G. Yoon, M. Lee, K. Kim et al., "Morphology and mechanical properties of multi-stranded amyloid fibrils probed by atomistic and coarse-grained simulations," Physical Biology, vol. 12, no. 6, Article ID 066021, 2015.

[35] J. Dong, C. E. Castro, M. C. Boyce, M. J. Lang, and S. Lindquist, "Optical trapping with high forces reveals unexpected behaviors of prion fibrils," Nature Structural and Molecular Biology, vol. 17, no. 12, pp. 1422-1430, 2010.

[36] A. W. P. Fitzpatrick, S. T. Park, and A. H. Zewail, "Exceptional rigidity and biomechanics of amyloid revealed by $4 \mathrm{D}$ electron microscopy," Proceedings of the National Academy of Sciences of the United States of America, vol. 110, no. 27, pp. 10976-10981, 2013.

[37] M. F. M. Engel, L. Khemtémourian, C. C. Kleijer et al., "Membrane damage by human islet amyloid polypeptide through fibril growth at the membrane," Proceedings of the National Academy of Sciences of the United States of America, vol. 105, no. 16, pp. 6033-6038, 2008. 
[38] S. E. Cross, Y.-S. Jin, J. Rao, and J. K. Gimzewski, "Nanomechanical analysis of cells from cancer patients," Nature Nanotechnology, vol. 2, no. 12, pp. 780-783, 2007.

[39] M. Tanaka, S. R. Collins, B. H. Toyama, and J. S. Weissman, “The physical basis of how prion conformations determine strain phenotypes," Nature, vol. 442, no. 7102, pp. 585-589, 2006.

[40] M. J. Buehler, S. Keten, and T. Ackbarow, "Theoretical and computational hierarchical nanomechanics of protein materials: deformation and fracture," Progress in Materials Science, vol. 53, no. 8, pp. 1101-1241, 2008.

[41] K. Eom, G. Yoon, J.-L. Kim, and S. Na, "Coarse-grained elastic models of protein structures for understanding their mechanics and dynamics," Journal of Computational and Theoretical Nanoscience, vol. 7, no. 7, pp. 1210-1226, 2010.

[42] K. Eom, Simulations in Nanobiotechnology, CRC Press: Taylor \& Francis Group, Boca Raton, Fla, USA, 2011.

[43] M. Sotomayor and K. Schulten, "Single-molecule experiments in vitro and in silico," Science, vol. 316, no. 5828, pp. 1144-1148, 2007.

[44] M. J. Buehler and S. Keten, "Colloquium: failure of molecules, bones, and the Earth itself," Reviews of Modern Physics, vol. 82, no. 2, pp. 1459-1487, 2010.

[45] J. Adamcik and R. Mezzenga, "Study of amyloid fibrils via atomic force microscopy," Current Opinion in Colloid and Interface Science, vol. 17, no. 6, pp. 369-376, 2012.

[46] K. F. Freed, "Functional integrals and polymer statistics," Advances in Chemical Physics, vol. 22, pp. 1-128, 1972.

[47] H. Yamakawa and M. Fujii, "Wormlike chains near the rod limit: path integral in the WKB approximation," The Journal of Chemical Physics, vol. 59, no. 12, pp. 6641-6644, 1973.

[48] S. Kumar and M. S. Li, "Biomolecules under mechanical force," Physics Reports, vol. 486, no. 1-2, pp. 1-74, 2010.

[49] T. Strick, J.-F. Allemand, V. Croquette, and D. Bensimon, "Twisting and stretching single DNA molecules," Progress in Biophysics and Molecular Biology, vol. 74, no. 1-2, pp. 115-140, 2000.

[50] A. K. Mazur, "Wormlike chain theory and bending of short DNA," Physical Review Letters, vol. 98, no. 21, Article ID 218102, 2007.

[51] C. Rivetti, M. Guthold, and C. Bustamante, "Scanning force microscopy of DNA deposited onto mica: equilibration versus kinetic trapping studied by statistical polymer chain analysis," Journal of Molecular Biology, vol. 264, no. 5, pp. 919-932, 1996.

[52] F. Pampaloni, G. Lattanzi, A. Jonáš, T. Surrey, E. Frey, and E.-L. Florin, "Thermal fluctuations of grafted microtubules provide evidence of a length-dependent persistence length," Proceedings of the National Academy of Sciences of the United States of America, vol. 103, no. 27, pp. 10248-10253, 2006.

[53] G. Voth, Coarse-Graining of Condensed Phase and Biomolecular Systems, CRC Press, 2008.

[54] A. J. Rader, "Coarse-grained models: getting more with less," Current Opinion in Pharmacology, vol. 10, no. 6, pp. 753-759, 2010.

[55] P. Sherwood, B. R. Brooks, and M. S. P. Sansom, "Multiscale methods for macromolecular simulations," Current Opinion in Structural Biology, vol. 18, no. 5, pp. 630-640, 2008.

[56] C. Hyeon and D. Thirumalai, "Capturing the essence of folding and functions of biomolecules using coarse-grained models," Nature Communications, vol. 2, article 487, 2011.

[57] M. M. Tirion, "Large amplitude elastic motions in proteins from a single-parameter, atomic analysis," Physical Review Letters, vol. 77, no. 9, pp. 1905-1908, 1996.
[58] A. R. Atilgan, S. R. Durell, R. L. Jernigan, M. C. Demirel, O. Keskin, and I. Bahar, "Anisotropy of fluctuation dynamics of proteins with an elastic network model," Biophysical Journal, vol. 80, no. 1, pp. 505-515, 2001.

[59] I. Bahar and A. J. Rader, "Coarse-grained normal mode analysis in structural biology," Current Opinion in Structural Biology, vol. 15, no. 5, pp. 586-592, 2005.

[60] I. Bahar, C. Chennubhotla, and D. Tobi, "Intrinsic dynamics of enzymes in the unbound state and relation to allosteric regulation," Current Opinion in Structural Biology, vol. 17, no. 6, pp. 633-640, 2007.

[61] F. Tama and C. L. Brooks III, "Symmetry, form, and shape: guiding principles for robustness in macromolecular machines," Annual Review of Biophysics and Biomolecular Structure, vol. 35, pp. 115-133, 2006.

[62] K. Eom, S.-C. Baek, J.-H. Ahn, and S. Na, "Coarse-graining of protein structures for the normal mode studies," Journal of Computational Chemistry, vol. 28, no. 8, pp. 1400-1410, 2007.

[63] H. Jang, S. Na, and K. Eom, "Multiscale network model for large protein dynamics," The Journal of Chemical Physics, vol. 131, no. 24, Article ID 245106, 2009.

[64] C. Atilgan, O. B. Okan, and A. R. Atilgan, "Network-based models as tools hinting at nonevident protein functionality," Annual Review of Biophysics, vol. 41, no. 1, pp. 205-225, 2012.

[65] L. Meirovitch, Analytical Methods in Vibrations, Macmillan, New York, NY, USA, 1967.

[66] J. M. Gere, Mechanics of Materials, Thomson Learning, 6th edition, 2003.

[67] A. Kis, S. Kasas, B. Babić et al., "Nanomechanics of Microtubules," Physical Review Letters, vol. 89, no. 24, 2002.

[68] S. P. Timoshenko, "LXVI. On the correction for shear of the differential equation for transverse vibrations of prismatic bars," Philosophical Magazine Series 6, vol. 41, no. 245, pp. 744-746, 1921.

[69] K. C. Neuman, T. Lionnet, and J.-F. Allemand, "Single-molecule micromanipulation techniques," Annual Review of Materials Research, vol. 37, pp. 33-67, 2007.

[70] D. P. Allison, P. Hinterdorfer, and W. H. Han, "Biomolecular force measurements and the atomic force microscope," Current Opinion in Biotechnology, vol. 13, no. 1, pp. 47-51, 2002.

[71] E. M. Puchner and H. E. Gaub, "Force and function: probing proteins with AFM-based force spectroscopy," Current Opinion in Structural Biology, vol. 19, no. 5, pp. 605-614, 2009.

[72] K. Eom, J. Yang, J. Park et al., "Experimental and computational characterization of biological liquid crystals: a review of singlemolecule bioassays," International Journal of Molecular Sciences, vol. 10, no. 9, pp. 4009-4032, 2009.

[73] I. Usov and R. Mezzenga, "Correlation between nanomechanics and polymorphic conformations in amyloid fibrils," ACS Nano, vol. 8, no. 11, pp. 11035-11041, 2014.

[74] M. R. Sawaya, S. Sambashivan, R. Nelson et al., "Atomic structures of amyloid cross- $\beta$ spines reveal varied steric zippers," Nature, vol. 447, no. 7143, pp. 453-457, 2007.

[75] H. J. Chang, I. Baek, M. Lee, and S. Na, "Influence of aromatic residues on the material characteristics of $\mathrm{A} \beta$ amyloid protofibrils at the atomic scale," ChemPhysChem, vol. 16, no. 11, pp. 2403-2414, 2015

[76] K. Eom, "Mechanical characterization of protein materials," in Simulations in Nanobiotechnology, K. Eom, Ed., chapter 7, pp. 221-270, CRC Press, Boca Raton, Fla, USA, 2013. 
[77] M. Lee, H. J. Chang, D. Kim et al., "Relationship between structural composition and material properties of polymorphic hIAPP fibrils," Biophysical Chemistry, vol. 199, pp. 1-8, 2015.

[78] B. Choi, S. W. Lee, and K. Eom, "Nanomechanical behaviors and properties of amyloid fibrils," Multiscale and Multiphysics Mechanics, vol. 1, no. 1, pp. 53-64, 2016.

[79] J. I. Kim, M. Lee, I. Baek, G. Yoon, and S. Na, “The mechanical response of hIAPP nanowires based on different bending direction simulations," Physical Chemistry Chemical Physics, vol. 16, no. 34, pp. 18493-18500, 2014.

[80] G. Lee, W. Lee, H. Lee et al., "Mapping the surface charge distribution of amyloid fibril," Applied Physics Letters, vol. 101, no. 4, Article ID 043703, 2012.

[81] N. Mizuno, U. Baxa, and A. C. Steven, "Structural dependence of HET-s amyloid fibril infectivity assessed by cryoelectron microscopy," Proceedings of the National Academy of Sciences of the United States of America, vol. 108, no. 8, pp. 3252-3257, 2011.

[82] M. Kawahara, M. Kato, and Y. Kuroda, "Effects of aluminum on the neurotoxicity of primary cultured neurons and on the aggregation of $\beta$-amyloid protein," Brain Research Bulletin, vol. 55, no. 2, pp. 211-217, 2001.

[83] D. Drago, M. Folin, S. Baiguera, G. Tognon, F. Ricchelli, and P. Zatta, "Comparative effects of A $\beta(1-42)$-Al complex from rat and human amyloid on rat endothelial cell cultures," Journal of Alzheimer's Disease, vol. 11, no. 1, pp. 33-44, 2007.

[84] P. Zatta, Metal Ions and Neurodegenerative Disorders, World Scientific, Singapore, 2003.

[85] S. Parthasarathy, F. Long, Y. Miller et al., "Molecular-level examination of $\mathrm{Cu}^{2+}$ binding structure for amyloid fibrils of 40-residue Alzheimer's $\beta$ by solid-state NMR spectroscopy," Journal of the American Chemical Society, vol. 133, no. 10, pp. 3390-3400, 2011.

[86] A. Abelein, A. Gräslund, and J. Danielsson, "Zinc as chaperonemimicking agent for retardation of amyloid $\beta$ peptide fibril formation," Proceedings of the National Academy of Sciences of the United States of America, vol. 112, no. 17, pp. 5407-5412, 2015. 

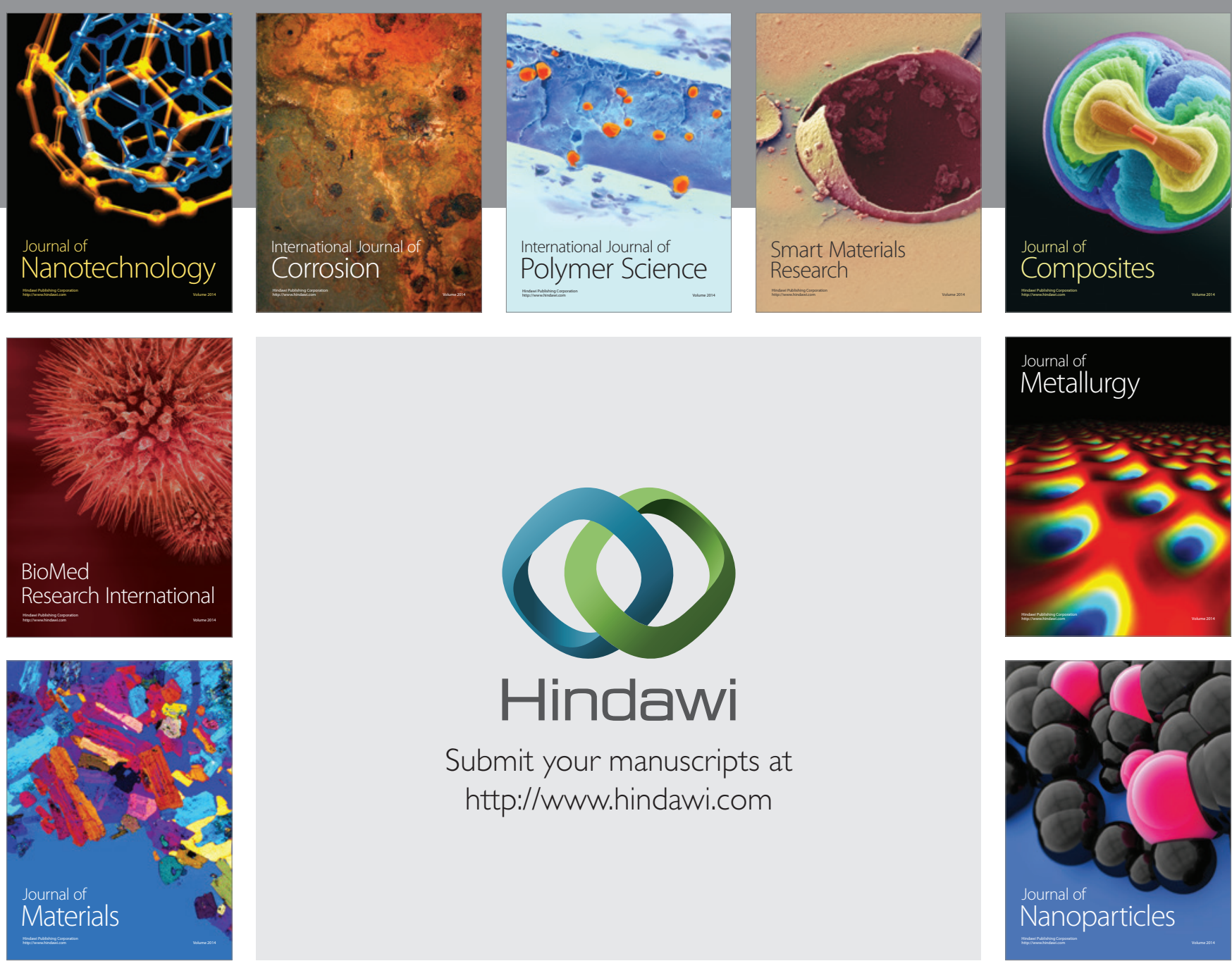

\section{Hindawi}

Submit your manuscripts at

http://www.hindawi.com

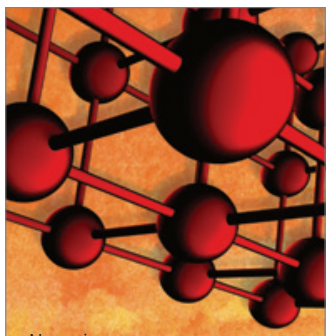

Materials Science and Engineering
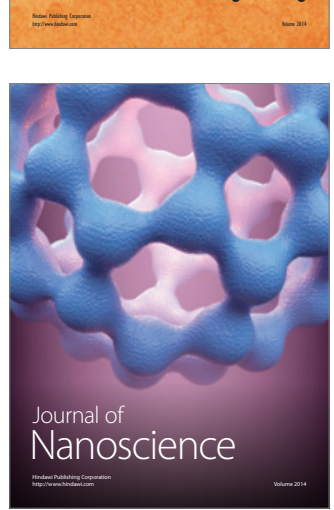
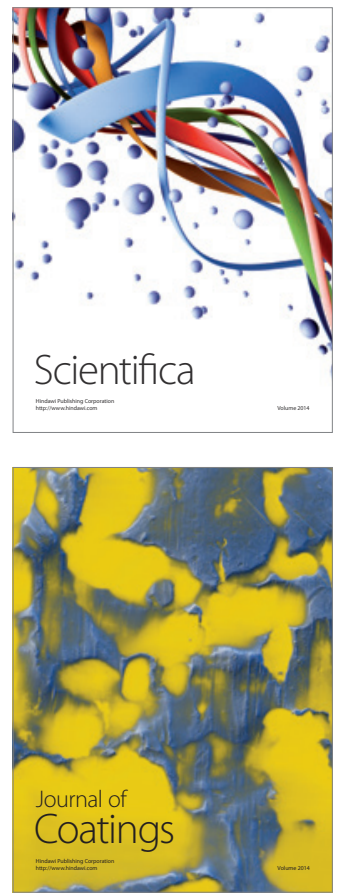
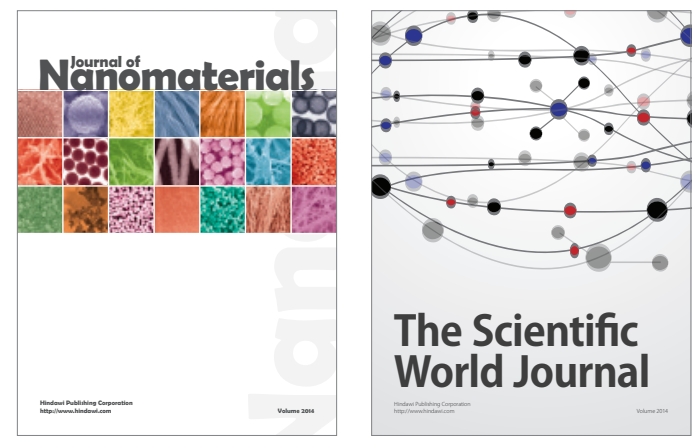

The Scientific World Journal
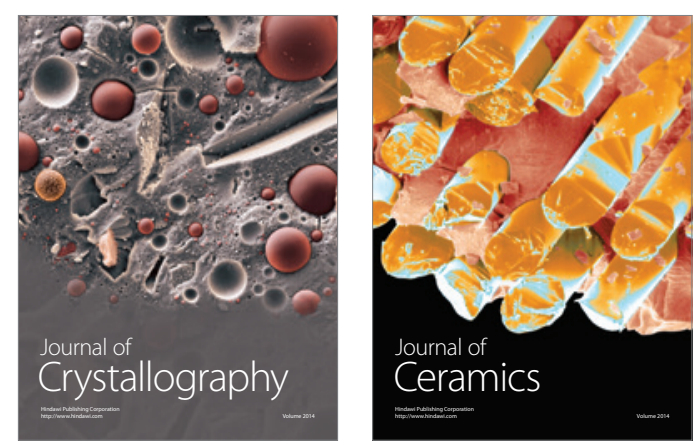
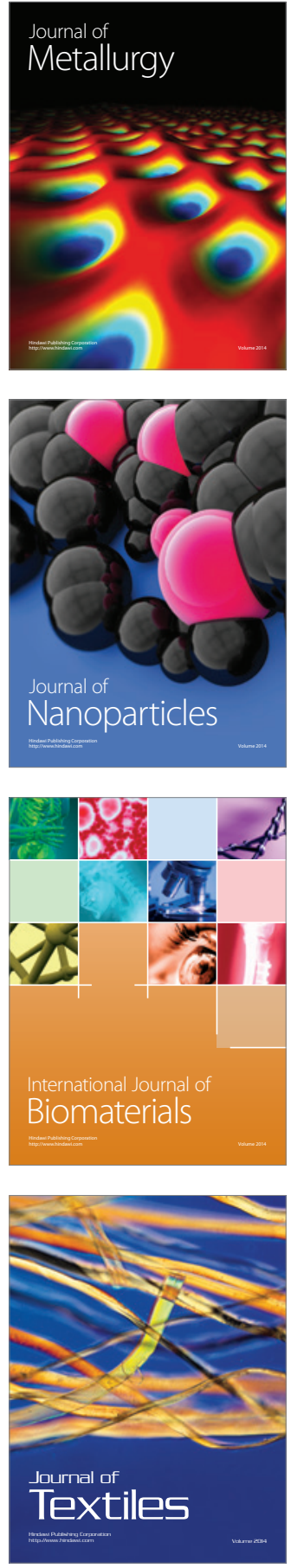